\title{
Biomechanical aspects in dental replacements
}

\author{
A. Baldini, G. Bruzzesi, D. Zaffe, M. Giacopini, A. Strozzi \& A. De Pol \\ University of Modena and Reggio Emilia, Italy.
}

\begin{abstract}
This chapter deals with biomechanical aspects in dental replacements. The state of the art is critically reviewed taking into account the body of the literature results. The initial section is devoted to the mechanical properties of bone and to a description of the jaw geometry and of its loading and constraining. The following section presents a classification of the various tooth replacement configurations and of the various materials adopted, where single and multiple replacements are considered. A specific section is devoted to the solid modelling of the jaw as input to numerical analyses, where the aid offered by reverse engineering and tomography is underlined. The finite element method as well as alternative numerical and experimental approaches are reviewed in a dedicated section. The stress analysis forecasts and measurements are biomechanically interpreted in the light of the current literature results. The chapter ends with a section devoted to biological aspects.
\end{abstract}

Keywords: dental implants, mechanical proprieties of bone, titanium, osseointegration, finite elements, cell method, boundary element, photoelasticity

\section{Introduction}

Over the last decades the use of endosseous dental implants for the restoration of totally or partially edentulous jaws has become a commonplace [1]. The basic idea is to replace the natural root of the tooth with a rigid foundation which will serve as an artificial root for the restored tooth. A typical prosthetic assembly based on this concept is exemplified in Figure 1. In the cases of extended edentulism, the implant can also be used as a base for a multiple-tooth prosthesis. The success of the implant overtime depends upon the degree of biofunctionality and biocompatibility it encompasses. Biofunctionality, which is mainly influenced by the geometric design of the implant, involves the response of the bone to the mechanical loads exerted by the prosthesis. If the induced stress falls within a suitable range, [2] new bone formation around the artificial root is favoured, thus promoting durable integration with the living tissue. By contrast, stress levels outside the optimal range (either too low or too high) result in bone resorption, causing failure of the implant. So, an optimal replacement design should favour a condition capable of promoting osseointegration. Biocompatibility, which primarily depends on the used material, is a measure of the biological affinity between the implant and the biological environment. The concept of osseointegration determines whether the tissue surrounding the implant will consist of high-strength cortical bone (implying full integration) or of low-strength trabecular bone (leading to poorer integration). This contribution is devoted to discussing the merits of modern dental implantology. 


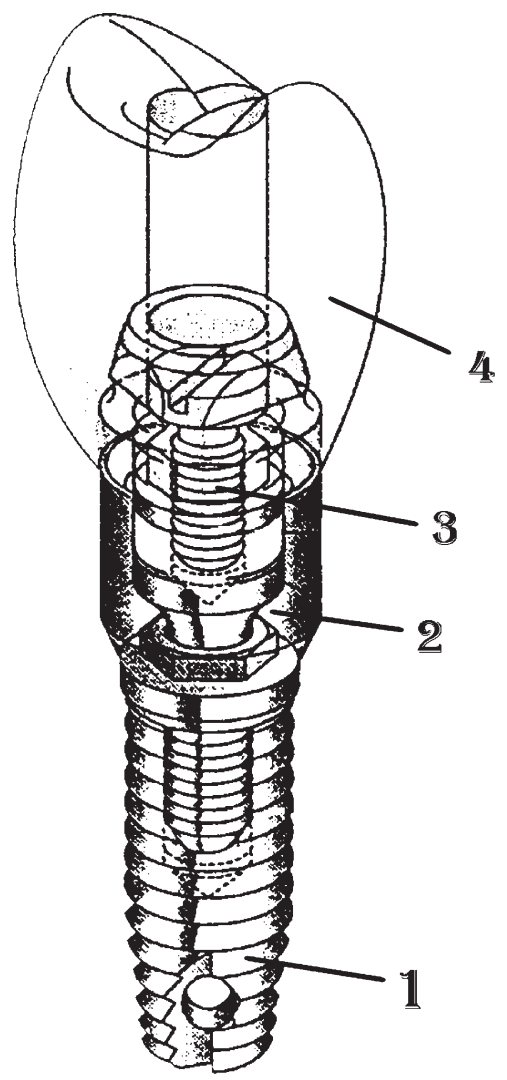

Figure 1: Typical prosthetic assembly.

\subsection{Mechanical properties of bone}

Bone is considered to be a porous material with complex microstructure. It is usually classified into cortical and trabecular tissue. It is recognised that the dense cortical bone has better load bearing capability than the highly porous trabecular bone. Upon insertion, cortical and trabecular bone tends to form an envelope about the implant, starting from bone surfaces and gradually forming a partial to complete integration. However, the degree of integration depends on the stresses generated and the location of the implant in the jaw. Usually, 100\% cortical osseointegration is recorded only in the anterior mandible, which decreases towards the posterior region. The least cortical osseointegration $(<25 \%)$ is recorded in the posterior maxilla. The degree of osseointegration appears to be a property of the quality of bone and the stresses developed. The stresses in turn depend on the implant geometry, implant material and loading. Since there is no control over the location of the implant, it is important to control stress levels with proper implant geometry and material. However, the requirement of biocompatibility often dictates the choice of material and the implant geometry is the only parameter that may be changed easily.

Experiments have shown that bone has a stress-strain relationship similar to many engineering materials and Hooke's law is applicable for a limited range of strains [3]. It is observed that the ultimate strength and strain in compression are larger than the corresponding values in tension, whereas the modulus of elasticity in tension is larger than that in compression. This difference 
is caused by the non-homogeneous, anisotropic, composite structure of bone, which also causes different strength values when the bone is tested in different loading conditions. Lotz et al. [4] analyzed the anisotropic mechanical properties of metaphyseal bone. Cowin [5] analyzed the properties of diaphyseal bone. Although both used the transverse isotropic model, differences emerged between the observed properties.

Lotz has used von Mises yield criterion for cortical bone and von Mises and Hoffman's yield criterion for trabecular bone. It was found that strains at failure predicted by von Mises' criterion do not correspond well with measured values, but yield and fracture were accurately predicted. Bone is also seen to display viscoelastic properties. Lakes and Katz [6] discussed various physical processes contributing to viscoelasticity of bone including thermoelastic coupling, piezoelectric coupling, motion of fluid in bone canals, inhomogeneous deformation in osteons, cement lines, lamellae, interstitium and molecular modes in collagen.

Changes in bone structure and architecture may take place slowly (months or years) due to the action of bone cells (osteoclasts for resorption, and osteoblasts for formation) or rapidly (days) due to uptake of output of mineral salts. Wolff [2] first advanced the idea that living bones change according to the stress and strain acting in them. Change in external shape is called surface remodelling. Change in porosity, mineral content and mass density is called internal remodelling. Evans [7] concluded that compressive stress stimulates the formation of new bone and is an important factor in fracture healing. Hert et al. [8] concluded that intermittent stress is a morphogenetic stimulus to functional adaptation and that the effect of compressive stress is the same as that of tensile stress. A detailed study of remodelling can be found in the writings by Fung [9].

Due to its biphasic structure, bone is much like fibreglass, with collagen fibres corresponding to the reinforcing glass fibres and bone matrix and hydroxyapatite crystals corresponding to the embedding resin matrix. Derived from the exhaustive measurements carried out by Reilly and Burstein [10], Knets [11] and Arendts and Sigolotto [12] on the human mandible, the elastic properties of jaw bones are collected in Table 1.

Concerning the mechanical strength of bones, the Tsai-Wu [13] criterion has been employed in Natali [14], but the more traditional von Mises equivalent stress has equally been employed.

\subsubsection{Bone resorption, deposition, remodelling, bone osseointegration and bone necrosis}

Some specific bone properties that gratify the doctor but terrify the engineer are described by the following terms: bone resorption (loss), bone formation (apposition, growth), bone remodelling, osseointegration and bone necrosis (death).

The existence of a relationship between the bone mechanical loading and its architecture was appreciated by Galileo, who is credited with being the first to understand the balance of forces in beam bending and applying this understanding to the mechanical analysis of bone.

Table 1: Elastic properties of jaw bone.

\begin{tabular}{|l|c|c|c|c|}
\hline & \multicolumn{2}{|c|}{ Young's modulus(GPa) } & \multicolumn{2}{c|}{ Poisson's ratio } \\
\hline Type of bone & Longitudinal & Transverse & $\begin{array}{l}\text { Longitudinal- } \\
\text { transverse }\end{array}$ & $\begin{array}{c}\text { Transverse- } \\
\text { longitudinal }\end{array}$ \\
\hline Cortical & $16-18$ & $6-10$ & 0.31 & 0.15 \\
\hline Trabecular & $0.2-0.5$ & 0.3 & \\
\hline
\end{tabular}


Bone remodelling is the structural and architectural adaptation of bone mass (or volume) in response to localised changes in loading conditions [15]. The remodelling of the cortical bone is particularly important, since the cortex sustains most of the load applied to the bone. (A paper addressing remodelling in connection to the cancellous bone adaptation is presented by Tsubota [16]). A major disturbing effect of bone remodelling is the possible loosening of replacements due to bone resorption, which in turn jeopardises the implant longevity. As a consequence, it would be highly desirable that predictive methods of determining changes in bone mass distribution be available.

Bone remodelling may imply loss or increase of bone mass, or even an equilibrium condition characterised by no net change of bone mass. It is believed that mechanical stress in living structures may be a trigger to induce bone remodelling. In other words, bone remodelling is assumed to be ruled by a stress-related signal, but the issue of which is the key variable and under what circumstances remains unresolved [15]. Among the candidate signals in the bone remodelling process, the tensile principal stress or strain, the hydrostatic stress component and the equivalent stress have been proposed. Unfortunately, the above stress indicators as remodelling parameters are strongly dissimilar. For instance, the tensile stress remodelling criterion is reminiscent of the equivalent stress suitable for estimating the strength of brittle materials, such as ceramics. Instead, the equivalent stress remodelling assumption reminds of the equivalent stress employed in strength calculations of metal components.

Despite the above signalled uncertainties, the tensile principal stress is generally credited as the best candidate signal in terms of the pattern of bone deposition and resorption.

Bone resorption is caused by low stress signals, that is low stress levels, often caused by a stress shielding effect. In other words, the implant, generally appreciably the bone stress distribution with respect to the physiological situation, possibly lowers the stresses in some bone zones. Thus the presence of such zones becomes unjustified from a load bearing viewpoint, and the skeletal segment reacts to this newly instituted stress distribution by promoting a resorption of the bone tissue in the zones where its presence becomes unnecessary, possibly up to complete disappearance. It may also be observed that, while in traditional structural mechanics the stress shielding, by lowering the stress level, constitutes a desired effect, in biomechanics it may entail negative aspects, since it may stimulate bone resorption, which in turn may cause an undesired implant loosening.

Bone formation and maturation is favoured by high stress levels, where the bone adapts its shape to the newly instituted stress distribution. The intermediate situation where the bone mass remains stable is named as equilibrium condition or dead zone. In some sense, bone remodelling tries to recover more or less the original stress state after the modifications caused by the implant insertion.

To represent graphically the bone remodelling processes, a trilinear curve has often been employed [15] where the remodelling signal is reported along the $x$-axis, whereas the growth stimulus is represented along the $y$-axis (Figure 2). Bone formation is stimulated when the remodelling signal reaches high values, whereas bone resorption takes place if the remodelling signal is low. The central dead zone represents a region of remodelling equilibrium under which there is no net change in bone mass.

Qualitative stress values for the various letters A, B, C, D and E defining transitional points in the diagram of Figure 2 are reported in the technical literature with great prudence. Usually point $\mathrm{C}$, defining the centre of the dead zone, is assumed to fall at about $75 \%$ of the physiological stress values [17]. The dead zone width may be assumed of the order of $40 \%$, that is, point lettered $\mathrm{D}$ is $20 \%$ to the right of point $\mathrm{C}$, whereas point $\mathrm{B}$ is $20 \%$ to the left of point $\mathrm{C}$. The normal range of bone strain is up to, say, $0.07 \%$, which in turn suggests physiological stresses in the 


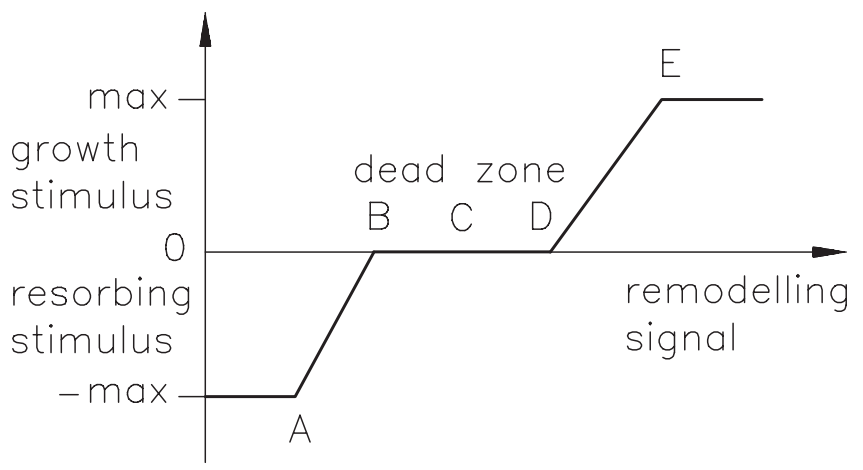

Figure 2: Bone remodelling processes.

cortical region of about $10 \mathrm{MPa}$. The position of points $\mathrm{B}$ and $\mathrm{D}$ may be estimated by proportions. The values associated to letters $\mathrm{A}$ and $\mathrm{E}$ remain the most difficult question.

The computer simulation is based upon an iterative approach. The stress field is computed for the bone initial shape. Then, the magnitude of the remodelling stimulus under the loading conditions is calculated from the remodelling signal using the trilinear curve of Figure 2. The value of stimulus defines the remodelling rate, and the resulting deposition or resorption is applied as a swelling strain perpendicularly to the cortical bone border. The stress distribution is then newly computed for the subsequent remodelling stage.

One particular difficulty in resolving which remodelling signals most closely match the clinical data is how to account for the loading history of the bone. In fact, remodelling is a result of fatigue stress cycles. If the loading cycle is not a stationary wave, as it always occurs in biomechanical problems, it is difficult to summarise its effects even in more traditional structural mechanics. Another aspect to carefully consider is the fact that computer forecasts have usually been obtained by adopting a linear elastic material, an approximation which may partially justify the disagreements encountered.

The qualitative rather than quantitative agreement between real remodelling and computer predictions has recently suggested the hypothesis that the bone adaptive process is limited to a certain finite post-operative period, and it terminates the bone remodelling even though the original stress state is not fully re-established [17].

Figure 2 suggests that a high stress level is beneficial, since it promotes bone growth. This conclusion cannot be true in general. For instance, in tooth implants it is known that peak stresses are detrimental, since they cause bone resorption. For instance, in Ref. [18], clinical evidence is reported according to which peri-implant bone loss is associated with oral implant overload. A strain of about $2.5 \%$ is reported as an estimate of the fracture limit, which would imply fracture stresses in the region of $300 \mathrm{MPa}$. It is generally believed that excessive stress levels produce bone fatigue micro-fractures [18], which possibly make the overloaded zone more deformable. As a consequence, this damaged zone is relieved from the highest stresses, so that bone resorption may take place. In conclusion, bone resorption occurs for too high or too low stresses. If the aim of computer predictions is to mimic only the first stage of implant history, a linear elastic modelling of the bone, ignoring bone remodelling, still retains some interest, since it indicates which bone portions will undergo bone remodelling.

Osseointegration means that the interface between the bone and the implant is assumed to be almost fully bonded. Brånemark is credited to have been the first to describe this bone bonding ability. Osseointegration may be promoted by special surface finishing or coating. 
Osseointegration of the implant is not instantaneous, but it occurs after a period of, say, a few months time. Usually, the healing period during which the dental implant remains unloaded is about 3-6 months [19]. The shear stress producing de-bonding of the osseointegrated interface between bone and root of a tooth implant subjected to torque has been measured to be about $4 \mathrm{MPa}$.

Bone necrosis means death of bone often by ischemia, that is, lack of blood irrigation.

Bone remodelling caused by orthodontic (corrective) tooth movements has been examined in Ref. [20]. Based on the experimental observation, that the deformations of the periodontal ligament are much higher than those of the alveolar bone, the geometry of natural tooth root and alveolus have been assumed constant in the numerical models. It has been found that the key stimulus initiating orthodontic tooth movements is the strain state within the periodontal ligament rather than the strain field within the alveolar bone. The importance of developing a suitable orthodontic remodelling law has been underlined.

\subsection{Jaw geometry, loading and supports}

Although this analysis is centred on the jay, to better understand the mechanical aspects of the whole chewing mechanism it is necessary to consider simultaneously the mandible, the jaw, and the denture (Figure 3). It is important to observe that, although both jaw and mandible pertain to the skull and play the same role, the morphology of the upper and lower dental arches differs appreciably. In fact, the structure of the mandible is tubular, where a cortical layer surrounds a trabecular core. The thickness of the cortical stratum varies from place to place, but every crosssection of the mandible exhibits a trabecular central part totally surrounded by a ring of cortical tissue. The mandible is a long bone, like the femur, but it is curved. The mandible is subjected to strains higher than their counterparts in the jaw. This fact is imputable to the circumstance that the mandible is a mobile bone with respect to the skull, and its mechanical properties must withstand the loads imparted by the masticatory muscles during a physiological activity, and by the occlusion exerted in a pathological occurrence, such as bruxism. Since the loads applied to the jaw during chewing are generally asymmetric, the jaw deflection may be noticeable.

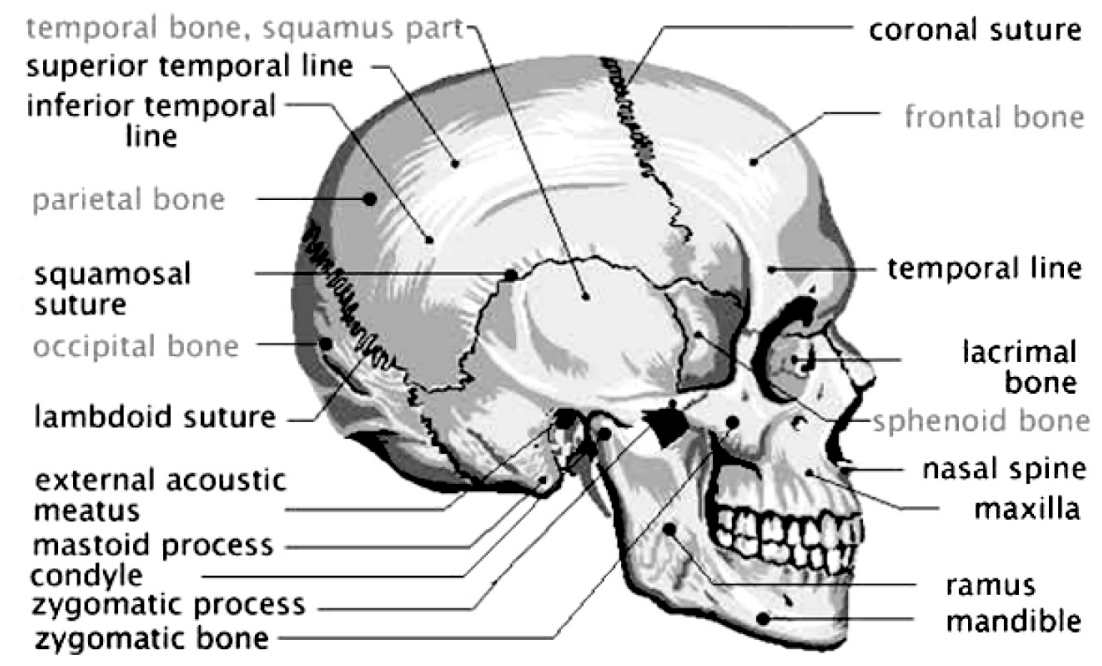

Figure 3: Skull bones. 
Unlike the mandible, which is an independent bone, the jaw is a continuation of the facial bones. The jaw exhibits a two-layer structure — cortical and trabecular — with double curvaturein width and height. The jaw cross-section is a half-ring of cortical bone containing a half-disc of spongy bone. As for the mandible, the thickness of the cortical stratum varies from zone to zone. Since the jaw is anchored to the skull base, the forces exerted by the masticatory muscles and by the teeth are partially transmitted to the skull bones through the canine and molar teeth. As a result of the above-commented load transfer mechanism favouring stress diffusion and limiting stress concentrations, the stresses affecting the jaw are lower than their analogues in the mandible.

The two mandible extremities are named condyles. Each condyle is inserted into a cavity of the temporal bone of the skull, where an articular disc is placed between the two mating surfaces. Figure 4 details the rotation axes of the condyles. The articular movements may occur along the three axes, namely horizontal, vertical and sagittal (or antero-posterior). Only the horizontal axis is common to the two condyles, and therefore it is also named transverse horizontal mandibular axis and, more rarely, bi-condylar axis.

The main movement of the mandible occurs as a result of a rotation about the horizontal axis; anyway, the various muscles acting on the masticatory articulation promote additional condylar rotations and translations. Rotations about one axis produce movements in the plane perpendicular to the axis being observed.

The muscles mainly involved in the chewing activity are positioned symmetrically with respect to the two sides of the mandible, and they are the masseter, the medial and lateral pterygoid and the temporalis [21] (Figure 5). A realistic modelling of the jaw mechanical behaviour requires the effects of the eight muscles mainly involved in the chewing activity to be accounted for.

The load intensities and directions are available in the pertinent literature [21]. In particular, the directions are expressed as direction cosines of the Cartesian reference system of Figure 5. For particulars the interested reader is invited to consult the original paper by Cruz et al. [21].

A thorough knowledge of the intensity, direction and load history is of the utmost relevance for the correct understanding of the biomechanical problems in the realm of oral implants. The forces applied to the dental arch during mastication may be resolved into the three directions of the axes of Figure 5. The vertical component is usually higher, and is better withstood by both the physiological dental system and by the implant. The forces may be either tensile or compressive; the latter are less dangerous, since they more rarely cause failure of the mechanical components. From a clinical viewpoint, however, it is highly unlikely that the loads

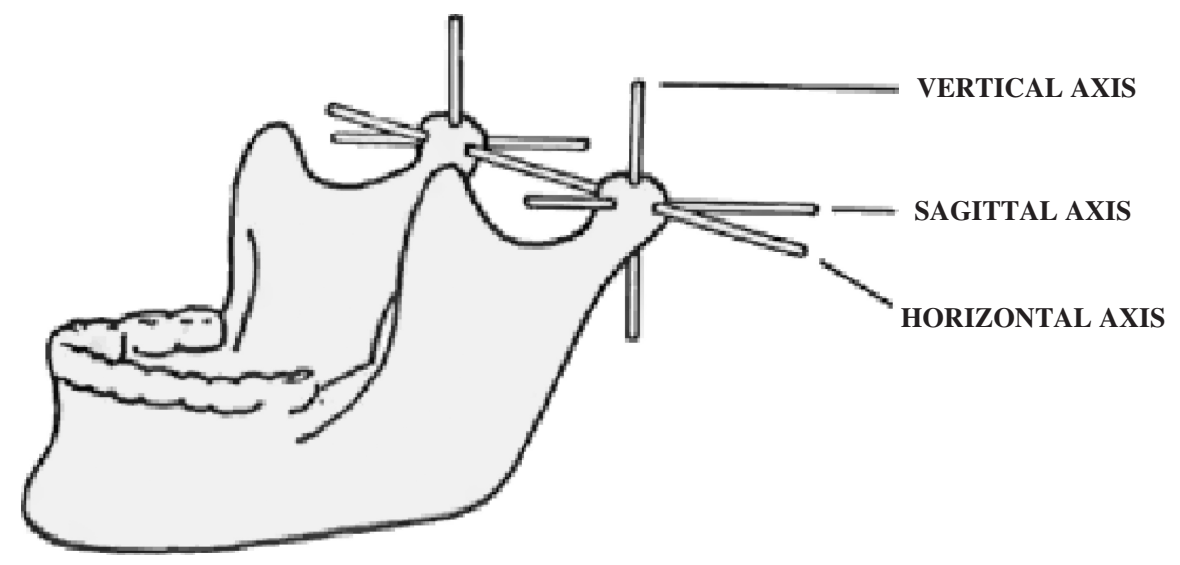

Figure 4: Rotation axes of the condyles. 


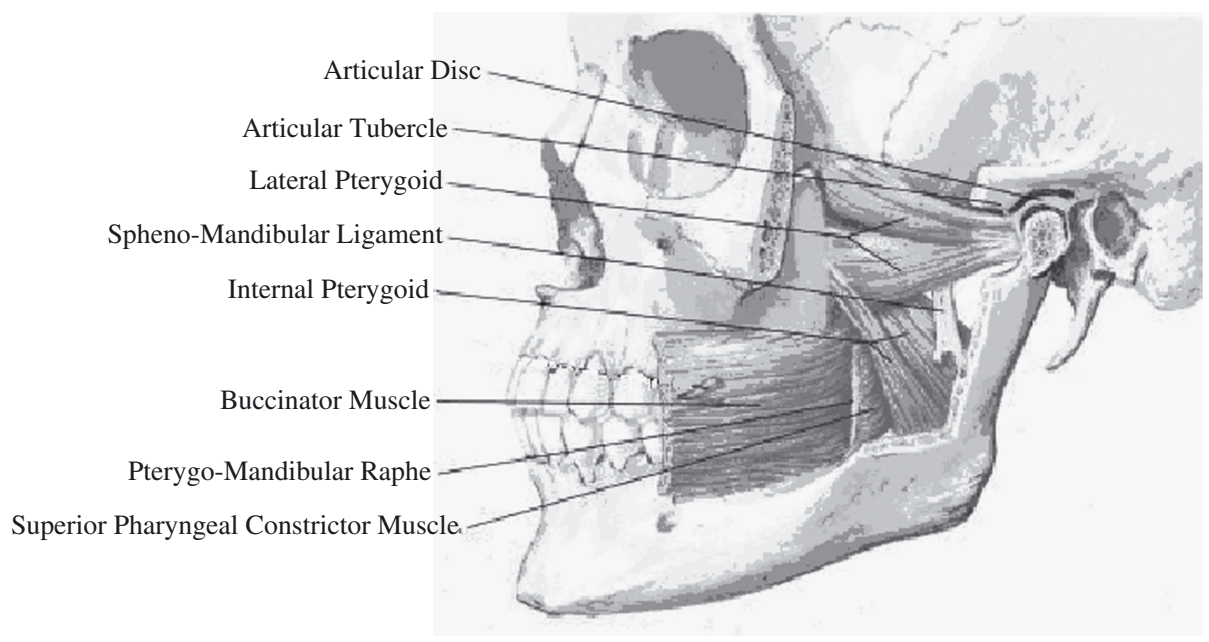

Figure 5: Muscles mainly involved in the chewing activity.

affecting the implant are purely in the direction of the tooth axis. Usually, the implant is subjected by a force offset with respect to the implant axis, or by additional transverse forces. As a consequence, the implant is simultaneously subjected to tension, bending and torque.

The stresses activated by the above loading are detrimental for the implant long-term prognosis. Their effects are negative with regard to (a) the interface between implant and bone, since they favour the bending and loosening of the mechanical components; and (b) the bone, which is damaged by the stress concentration responsible for bone resorption. Contrary to forces in the tooth axial direction, which produce stresses evenly distributed along the whole implant surface, a more complex force loading causes stresses unevenly concentrated at the crown and apex zones.

Studies carried out by various researchers concur in underlining that chewing loads may reach high values, especially those exerted by the posterior teeth. Such load intensities may cause mechanical difficulties, such as failure, or biomechanical problems, such as loss of osseointegration.

The forces applied to the implants may be either static or dynamic. The first category includes forces ascribable to para-functional habits, such as bruxism, and by stresses caused by dimensionally inaccurate implants. The second group encompasses all the chewing forces, from the regular ones up to those imputable to tooth impact against a rigid body. The static loads are often potentially detrimental, since their intensity may be particularly high. On the other side, one million of chewing cycles may be carried out per year, so that the dynamic forces due to physiological chewing may cause fatigue failure of the implant. According to many researchers, the most detrimental situation is the impact of the tooth against a rigid body, which entails high implant failure hazard.

The highest forces applied to the teeth act perpendicular to the occlusal plane (the imaginary surface on which upper and lower teeth meet) on the posterior teeth. The maximum occlusal force depends on the state of the dental arch and of the chewing muscles. For the natural teeth the load intensity during physiological chewing may reach $500 \mathrm{~N}$, and the time of application of the loads is very limited, about 9 min per day.

In addition to the physiological chewing forces, para-functional forces must be considered. Such occlusal forces are imputable to tooth shape, epilepsy and mental stress, and they may be intentional or unintentional. Pathological para-functions such as bruxism may produce forces as high as $2000 \mathrm{~N}$ and, consequently, they may be extremely detrimental for the chewing system. 


\section{Various kinds of tooth replacements}

A possible classification of dental implants is based on the biological interaction established with the bone. On this ground, the implants are divided in fibro (osseo) encapsulated and osseointegrated devices. The steel implants are usually of the first kind. Titanium or titanium alloy implants, by stimulating the formation of new bone, belong to the second category.

Osseointegrated titanium implants were first introduced by Brånemark [1] in the sixties. More recently, fully ceramic or ceramic-coated implants have appeared, which are claimed to further improve on the titanium competitors [22].

Within each of the two categories described above, dental implants are classified according to their shape. The richest variety of configurations is to be found among the fibro (osseo) encapsulated implants. Plates (typically supporting several teeth), discs (resting on the cortical bone of the jaw), an assortment of screws and many others are commonly used. On the other side, the osseointegrated implants basically subdivide according to the scale of bone apposition they exploit. This can happen on either a macroscopic or a microscopic level. Macroscopically-osseointegrated implants promote the mechanical interlocking of the bone around the macrogeometry of the part in the form of threads, holes or undercuts. Microscopically-osseointegrated implants seek an interconnection between the bone and the implant surface by means of the increased porosity of the latter. Plasma spraying and investment casting techniques are the usual processing methods employed to this purpose. The overall configuration of a mandibular tooth restored by means of a macroscopically-osseointegrated implant is shown in Figure 6. The essential components are the fixture (pillar) 1, the abutment and prosthetic screws 2 and 3 and the prosthetic crown 4 .

The crucial part of the implant is represented by the pillar, which embodies the artificial root of the restored tooth. The pillar is received into a hole drilled through the cortical surface layer well within the cancellous core of the jaw. The cancellous bone is unable to sustain significant stresses by itself, but can promote the formation of fibrous or cortical bone which possesses considerable mechanical strength. A radiograph of implanted pillars is shown in Figure 6, while Figure 7 displays the threaded opening of one such pillar surfacing from the jaw. Depending on the anatomic proportions of the patient and on the particular location of the jaws receiving the prosthesis, the length and the cross-section of the implanted pillar vary from

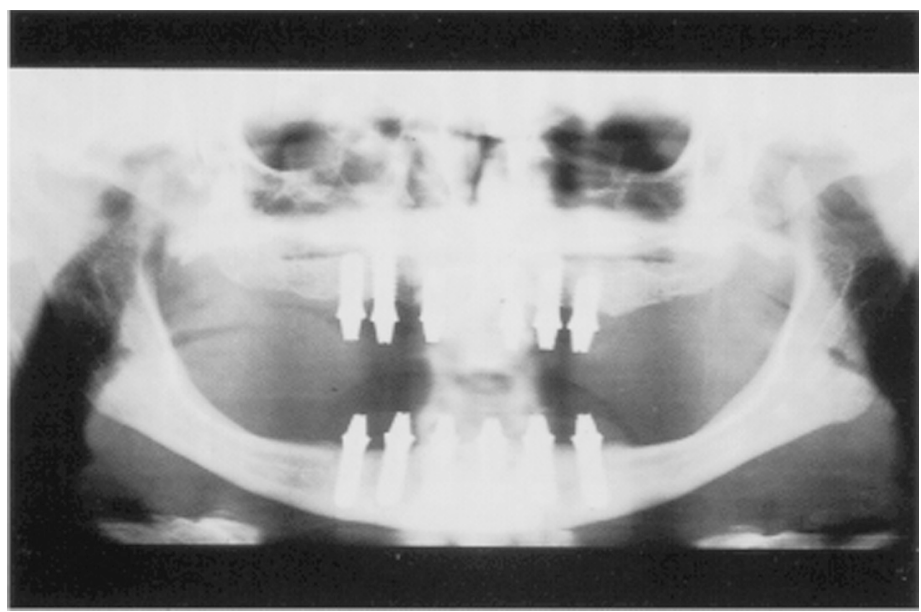

Figure 6: Radiograph of implanted pillars. 


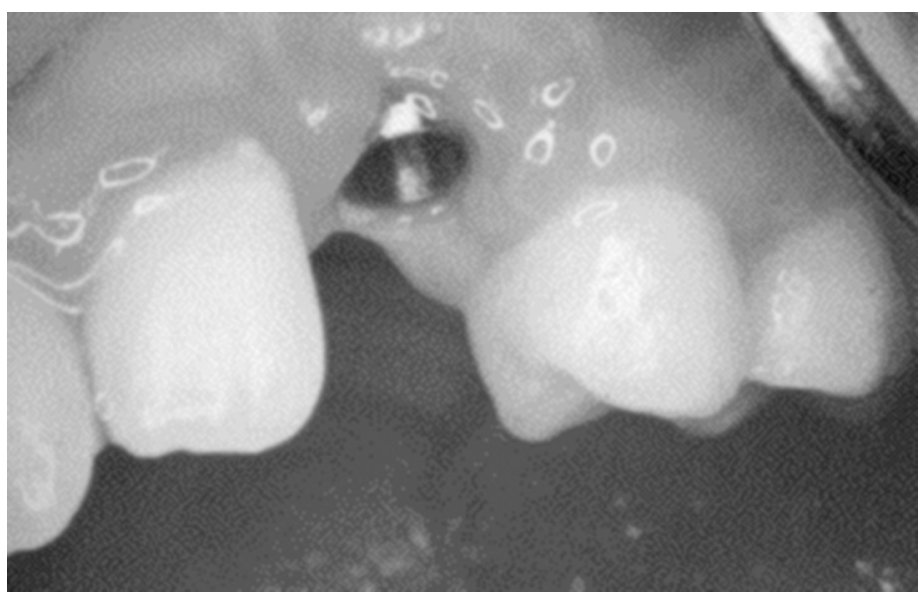

Figure 7: Threaded opening of one pillar.
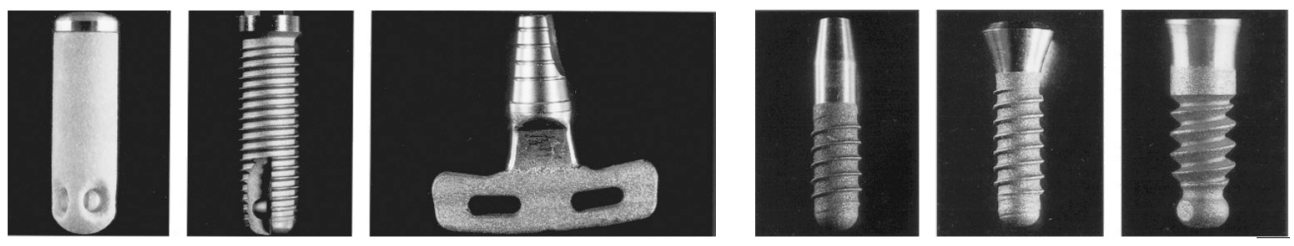

Figure 8: Implants.

case to case. An important modification to the basic design of Figure 1 is the incorporation of a shock absorber in the superstructure fitted to the fixture [23]. The function of the shock absorber is to replace the load dampening action of the periodontal ligament which is removed prior to implantation. An inevitably incomplete list of trade names of endosseous fixtures (with a concise indication of the materials and geometries) is given below:

1. Ankylos (uncoated pure titanium screw);

2. Brånemark (titanium screw);

3. BUD (titanium screw);

4. Calcitek (titanium cylinder coated with a hydroxyapatite film);

5. Dentsply (titanium screw or cylinder with or without hydroxyapatite coating);

6. 3 i (titanium alloy screw or cylinder);

7. Frialit 2 (pure titanium multistep cylinder with hydroxyapatite coating);

8. HA-TI (pure titanium conical screw);

9. Impla-B.I.T. (sandblasted pure titanium screw or lamina);

10. IMZ (plasma-flamed pure titanium cylinder);

11. ITI (plasma-flamed pure titanium screw);

12. Pit-Easy (plasma-sprayed pure titanium conical screw);

13. Steri-Oss (pure titanium screw or cylinder with or without hydroxyapatite coating);

14. ZL-Duraplant (pure titanium screw or cylinder).

A selection of the above-mentioned implants is displayed in Figure 8. 


\subsection{Tooth replacement materials}

The favourite materials for the construction of dental implants belong to the following categories:

1. Metals (pure titanium, titanium alloys, tantalum, stainless steel, $\mathrm{CoCr}$ alloys, etc.);

2. Ceramics (aluminium oxide (alumina), calciumphosfate, hydroxyapatite, etc.);

3. Composites (titanium and hydroxyapatite, alumina and hydroxyapatite, etc.).

The majority of the manufacturers prefer titanium and its alloys, both coated and uncoated. Titanium and titanium alloys exhibit an ultimate strength in the range of 400-800 $\mathrm{MPa}$, a Young's modulus of $110,000 \mathrm{MPa}$, and a strain at fracture of $20-30 \%$. The strength of a titanium alloy is therefore comparable to that of an average steel, whereas the Young's modulus is about half. This low value of the Young's modulus is beneficial to the implant because, being closer to the Young's modulus of the bone, limits the outcome of stress concentrations. The main reason why titanium has become so widespread is its high biocompatibility in comparison with other metals.

Even better than titanium in terms of biocompatibility are ceramic materials which, conversely, are often too brittle for structural applications. A felicitous combination between the best features of both materials consists in coating a tough titanium core with an inert ceramic layer. In addition, the natural porosity of ceramics favours ingrowth of the bone, thus contributing to an excellent microscopical osseointegration. This combination follows a modern trend in implantology, according to which any contact between metal and its local environment is avoided. Typical values of mechanical properties of compact ceramics are listed in Table 2 [22]. It appears that alumina, the most widespread ceramic in biomechanical applications, can reach an ultimate strength as high as that of most metals, although their toughness is much worse. The elongation at fracture of these compounds is always far below $1 \%$.

Ferrous alloys, although the first materials to have been used for dental implants, are becoming less and less popular, essentially because of the outcome of fibrous tissue instead of cortical bone around the implant.

Among the ferrous alloys, the most diffused material is stainless steel, with a tensile strength up to $1,000 \mathrm{MPa}$, a Young's modulus of $220,000 \mathrm{MPa}$, and an elongation at fracture of about $10 \%$.

Table 2: Physical and mechanical properties of pure ceramics.

\begin{tabular}{|l|c|c|c|c|c|c|}
\hline \multicolumn{1}{|c|}{ Material } & $\begin{array}{c}\text { Specific } \\
\text { gravity }\end{array}$ & $\begin{array}{c}\text { Young's } \\
\text { modulus } \\
(\mathrm{GPa})\end{array}$ & $\begin{array}{c}\text { Poisson's } \\
\text { ratio }\end{array}$ & $\begin{array}{c}\text { Tensile } \\
\text { strength } \\
(\mathrm{MPa})\end{array}$ & $\begin{array}{c}\text { Compressive } \\
\text { strength } \\
(\mathrm{MPa})\end{array}$ & $\begin{array}{c}\text { Hardness } \\
\text { (knoop) }\end{array}$ \\
\hline Alumina & $3.8-4.0$ & $380-410$ & 0.27 & $270-500$ & $3,000-5,000$ & 20,000 \\
\hline Zirconia & $5.6-5.9$ & $195-210$ & 0.27 & $500-650$ & - & 17,000 \\
\hline $\begin{array}{l}\text { Silicon } \\
\text { carbide }\end{array}$ & $3.1-3.2$ & $350-450$ & 0.27 & $250-600$ & 650 & 27,000 \\
\hline
\end{tabular}




\subsection{Single replacement}

The single replacement is usually constituted by four parts, namely (a) the ceramic crown, (b) the abutment, (c) the abutment screw and (d) the fixture or implant. The fixture is inserted into the bone, and it is also named bone screw or root. The abutment screw fixes the abutment to the implant. The ceramic crown is connected to the abutment. Figure 9 illustrates the above four components.

The advantage of a non-monolithic replacement is that, in the case of mechanical failure or yielding of the replacement, or of excessive wear of the crown, the components (a), (b) and (c) may be substituted without explanting part (d). The disadvantage of a dismountable replacement is its complexity and cost. Another problem frequently signalled is an undesired loosening of the abutment screw [24, 25]. Consequently, the major critical aspects concerning the assembly of a dismountable replacement are the stability of the screwed connection and the structural analysis of the stress field and contact pressure among the various mechanical parts. A study dealing with stability problems is that of Ref. [26], where the effects of a conical fitting are examined.

The consequence of a conical implant-abutment interface is further examined in Ref. [27]. A second critical aspect is the evaluation of the contact stresses at the implant-bone interface, and particularly the stresses within the bone. In fact, excessive stresses at the contact between implant and bone may cause an undesired bone resorption. More precisely, it is generally believed that bone loss is the result of bone remodelling secondary to bone micro-damage. In the light of this view, a dental implant should be designed such that the peak bone stresses are minimised. However, a series of experiments have indicated that a bone loss may also be a consequence of insufficient mechanical stimulation of the bone [27].

The physiological values of the mastication load are of the order of $100 \mathrm{~N}$ for the axial force and of $20 \mathrm{~N}$ for the lateral force [28], but the load may reach values as high as $500 \mathrm{~N}$ [29]. Both the stresses at the bone-implant interface and those imputable to the contact among the various mechanical parts usually increase with load inclination, where such increase appreciably depends

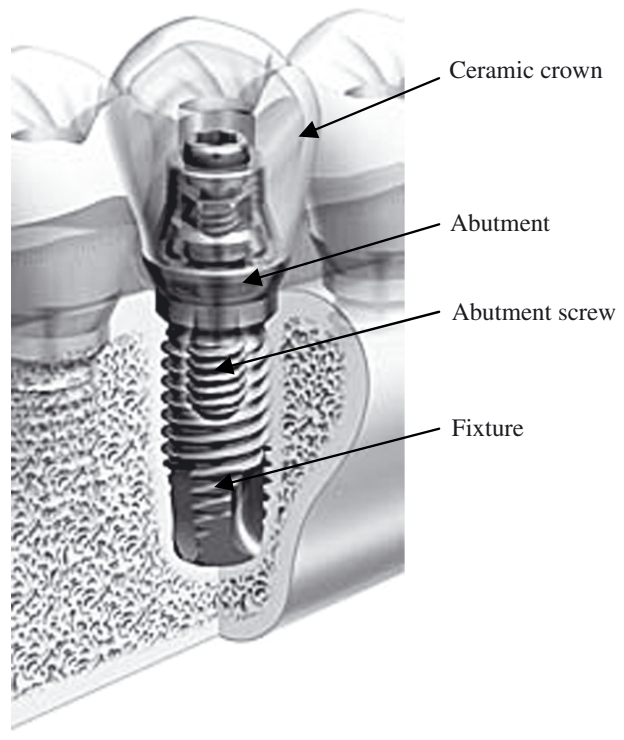

Figure 9: Replacement components. 
Table 3: Tensile strength, yield (Rs).

\begin{tabular}{|l|l|}
\hline ASTM Titanium Grade 1 & $170 \mathrm{MPa}$ \\
\hline ASTM Titanium Grade 2 & $280 \mathrm{MPa}$ \\
\hline ASTM Titanium Grade 3 & $380 \mathrm{MPa}$ \\
\hline ASTM Titanium Grade 4 & $480 \mathrm{MPa}$ \\
\hline
\end{tabular}
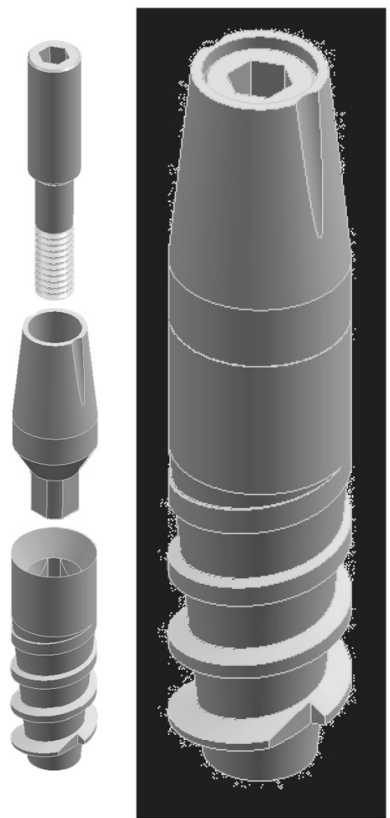

Figure 10: Virtual replacement.

upon the implant position relative to the mandible. Stress reduction is achieved by positioning the implant so that the load remains as axial as possible during mastication.

Due to biocompatibility, the fixture is made of titanium alloy grade 3 or 4 . The grade is an index of the titanium purity, that is of its biocompatibility, but it also appreciably affects the mechanical properties of titanium, as reported in Table 3.

It appears from Table 3 that the peak stresses within the tooth implant reach the highest titanium yield values so that a local yielding is unavoidable, although it is usually limited to restricted portions of the replacement. If a limited yielding may help prevent loosening of the abutment screw, it may produce a shortening of the fatigue life of the component. It is therefore necessary to resort to a replacement design constituting a trade-off between stability and strength needs.

Figure 10 displays a typical commercial replacement. The contact profiles have been redesigned in order to limit the contact stresses. The initial blocking torque applied to the abutment screw has been assumed to be $35 \mathrm{~N}-\mathrm{cm}$ [26]. The 3D finite element model employed to assess the contact stresses is illustrated in Figures 10-12, which clarifies the location of the stress peaks. As usual, high stresses occur at the screw first threads, zone A, and at the extremities of the implant-abutment contact, zone B, and of the abutment-abutment screw contact, zone C. 


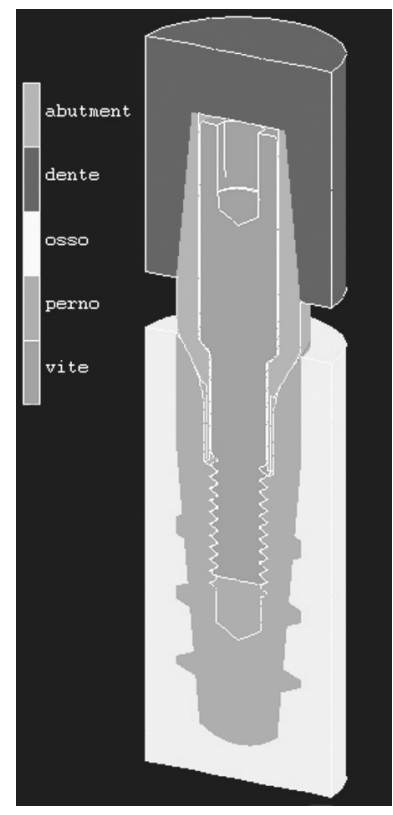

Figure 11: FE study.

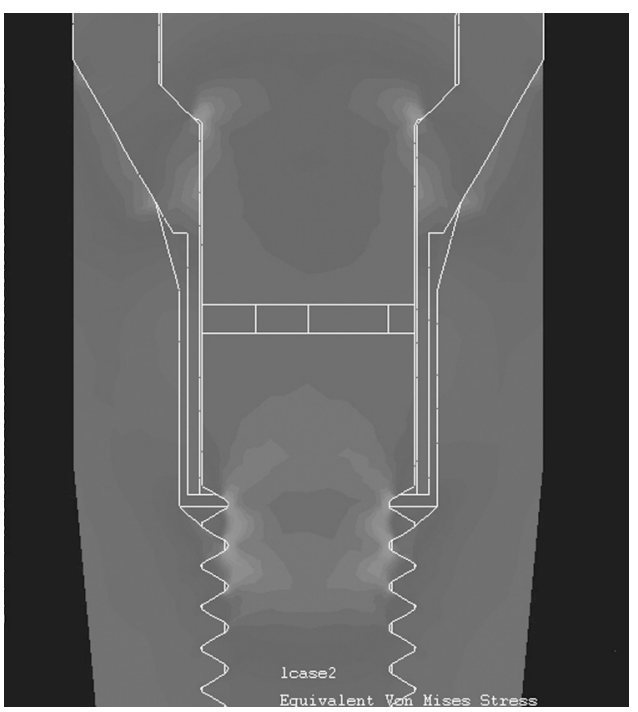

Figure 12: FE stresses.

To reduce the screw stresses, zone A, the screw geometry may be modified by stress shielding the thread with a groove, as in Figure 13. In addition, a variable pitch thread may be adopted in order to evenly distribute the forces between male and female, thus lowering the stresses in the vicinity of the first threads [30-36]. The pitch variation is of the order of some micrometres, which appreciably increases the implant cost. A stress reduction of about $30 \%$ is feasible. Figure 13 shows that the geometrically modified screw attains more uniform WIT Transactions on State of the Art in Science and Engineering, Vol 35, C 2008 WIT Press 


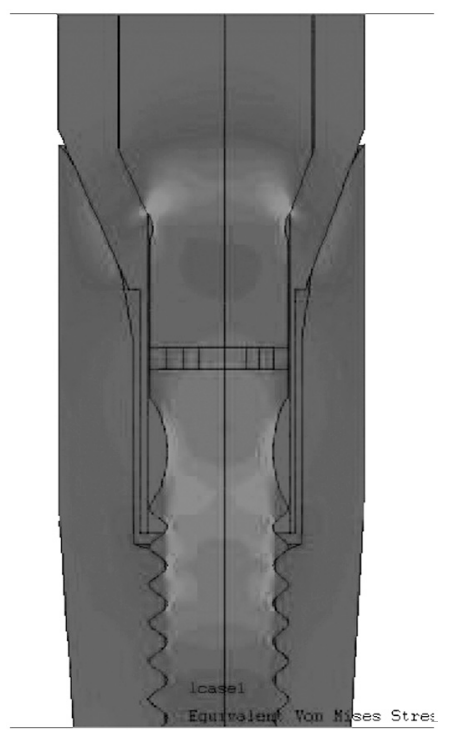

Figure 13: FE stresses.

stresses in the screw zone. It must however be remembered that the stress reduction attainable with a screw pitch variation unfortunately depends on the load exerted. In other words, for different loads the optima pitch variation changes.

Moving to the stress concentrations at the extremities of the implant-abutment contact, zone $\mathrm{B}$, and of the abutment-abutment screw contact, zone $\mathrm{C}$, appreciable improvements may be achieved by re-designing the contact profile to more evenly distribute the contact pressure. The use of proper fillets at the contact extremities is extremely beneficial [37].

\subsection{Multiple replacement}

An endosseous oral implant alternative to the single replacement is the multiple replacement. In the single replacement, every implant corresponds to a single tooth, whereas in a multiple replacement the dental arc rests on a horseshoe-like cantilever, which is sustained by a limited number of implants into the mandible. With respect to single replacements, the multiple replacement solution limits the number of implanted roots, and therefore it is preferred in the case of extended or total replacement of the dental arc. Four to seven roots are usually implanted to sustain the multiple replacement, whereas a maximum number of 12 adjacent single implants have been used to reconstruct the dental arc.

Figures 14 and 15 show the reconstruction of a whole dental arc possibly lost as a consequence of a traumatic event. A reconstruction of a part of the dental arc may also be effected with such multiple replacements, as in Refs. [38, 39].

The first problem encountered in the mechanical analysis of multiple replacements is the determination of the forces loading the various roots, an information which is of the utmost relevance in the multiple replacement design. Of importance is not only the maximum compression force exerted on a root, but also the value of the tensile force affecting the root. In fact, contrary to single replacements, the roots of multiple replacements may be affected by tensile forces too.

In fact, unlike single replacements, where the applied load is the local mastication force, the mechanical structure of multiple replacements is technically a statically redundant structure, WIT Transactions on State of the Art in Science and Engineering, Vol 35, C 2008 WIT Press 


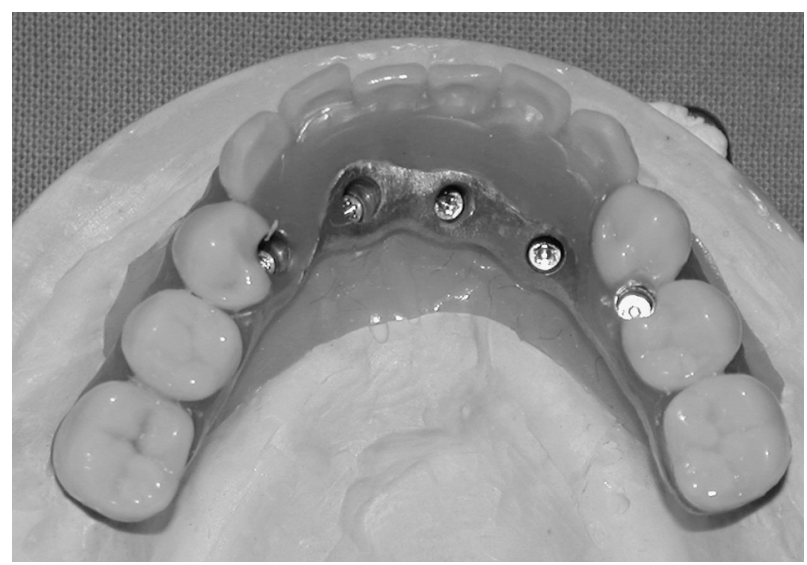

Figure 14: Reconstruction.

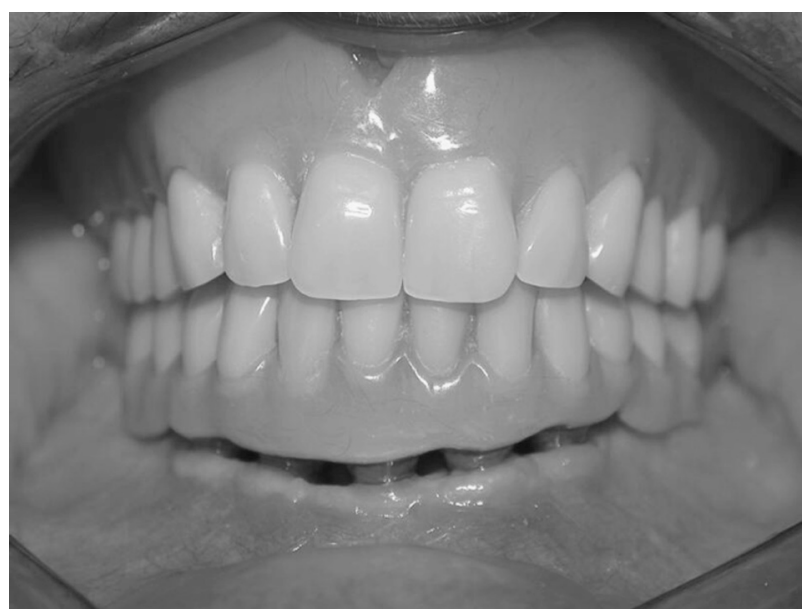

Figure 15: Reconstruction.

which implies that the percentage of the total load applied to the implant is distributed among the various roots in a complex way. More technically, the total load distribution cannot be computed from simple equilibrium equations, but the flexibility of the mandible and cantilever must be properly accounted for.

Since a realistic model would be too complex for everyday applications, an approximate analytical model has been proposed for the computation of the forces applied to the various roots [40]. This model is based upon appreciable simplifications of the system composed by the multiple replacement and mandible. More exactly, the cantilever and mandible are assumed as rigid, while the only deformable elements are the cantilever supports (Figure 16a). A similar assumption has been adopted in classical structural mechanics with regard to the calculation of a flange fixed to a support with screws.

Figure 17 illustrates the limits of the Skalak model. Figure 17(a) presents a sketch of the cantilever supported by five roots, in its unloaded state. Figure 17(b) shows the Skalak assumption, 


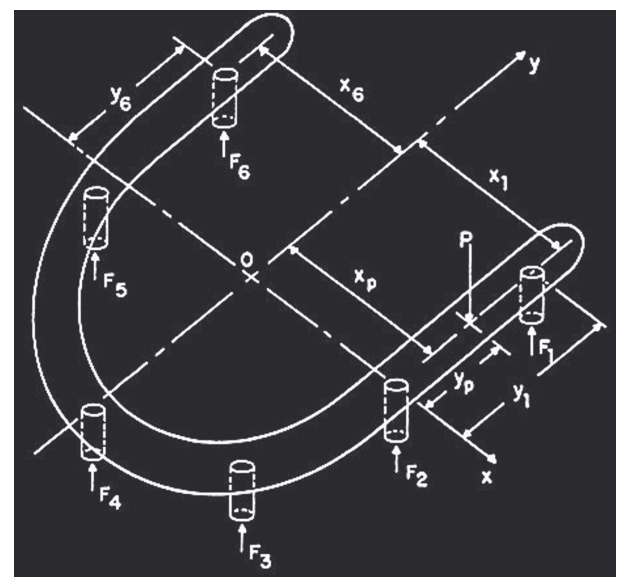

(a)

$$
\begin{aligned}
& F_{i}=\frac{P}{N}+P \cdot\left(A \cdot x_{i}+B \cdot y_{i}\right) \\
& A=\frac{\left(I_{x y} \cdot y_{p}-I_{x x} \cdot x_{p}\right)}{I_{x y}^{2}-I_{x x} I_{y y}} \quad B=\frac{\left(I_{x y} \cdot x_{p}-I_{y y}, y_{p}\right)}{I_{x y}^{2}-I_{x x} I_{y y}} \\
& I_{x x}=\sum y_{i}^{2} \quad I_{y y}=\sum x_{i}^{2} I_{x y}=\sum x_{i} y_{i}
\end{aligned}
$$

(b)

Figure 16: (a) Skalak model, (b) Skalak equations.

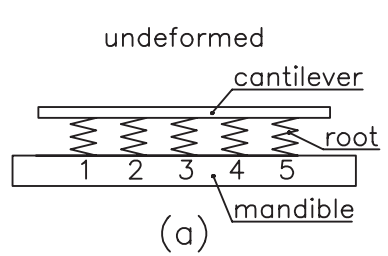

(a)

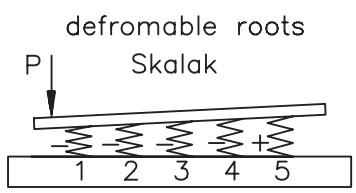

(b)

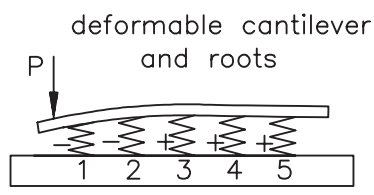

(c)

Figure 17: Limits of the Skalak model.

where the cantilever behaves as rigid. The application of a force $\mathrm{P}$ in the cantilever zone projecting beyond the extreme root produces a rotation of the cantilever, which in turn compresses roots 1 to 4 and applies a tensile force to root 5. Figure 17(c) displays the results of a more realistic model, where the cantilever and roots are both deformable. The compression load exerted on root 1 is much higher than the corresponding value according to the Skalak model, and the tensile force acting on root 3 is stronger than the unique tensile load affecting root 5 in the Skalak model. In conclusion, the Skalak model generally underestimates both the maximum compression forces and the maximum tensile loads acting on the roots. Such limits have been lucidly signalled in the Skalak contribution [40]. With the above simplifications, the loads affecting the various roots may be easily evaluated with the formulae given by Ref. [40] and reported in Figure 16(b).

In Ref. [41] the cantilever collapse is assumed to occur with compression loads higher than $200 \mathrm{~N}$, and with tensile loads higher than $400 \mathrm{~N}$. With modern materials, higher threshold values may be adopted.

The position of the roots affects the stress distribution within the cantilever, and also the force distribution among the sustaining roots. Cantilevers with appreciable portions projecting beyond the last root may exhibit prohibitively high stresses.

Finite element models of multiple replacements have been carried out in Ref. [42], to assess the force distribution among the supporting roots. To get realistic results, it is necessary to thoroughly model the various components. As a consequence, tomography is the most suitable method to get a faithful virtual model of the mandible. 
The solid modelling of a multiple replacement is presented in Figure 18, which adopts seven roots. A plaster moulding of the actual bone surface has also been employed to better define the implant zones (Figure 19).

The finite element mesh developed to perform a finite element analysis is presented in Figure 20.

The total load applied to the multiple replacement differs appreciably depending on whether the normal mastication or pathologies are modelled. In fact, in the case of bruxism the applied force may reach values as high as ten times the normal values. As a consequence, the multiple replacement, which stands on a limited number of roots, cannot be implanted in patients affected by bruxism.

The loads applied to the mandible extremities are discussed in Refs. [21, 43-45]. The forces applied by the muscles are shown in Figure 21. The muscles transmitting forces to the mandible are the masseter $(\mathrm{M})$, medial pterygoid $(\mathrm{Pm})$, lateral pterygoid $(\mathrm{Pl})$ and temporalis $(\mathrm{T})$. The corresponding forces may be evaluated from the muscle cross-sections following Ref. [46].

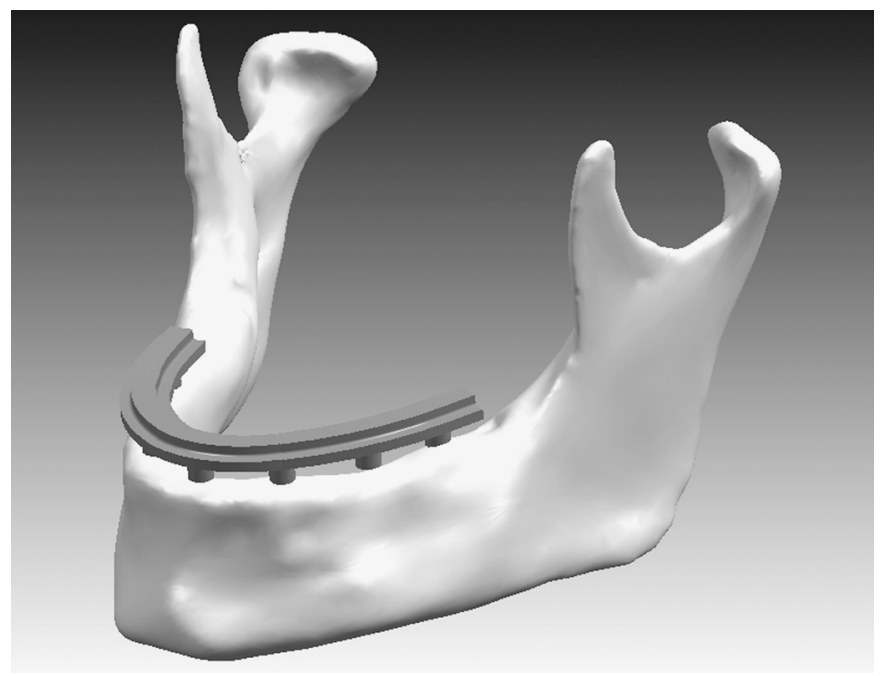

Figure 18: Solid modelling of a multiple replacement.

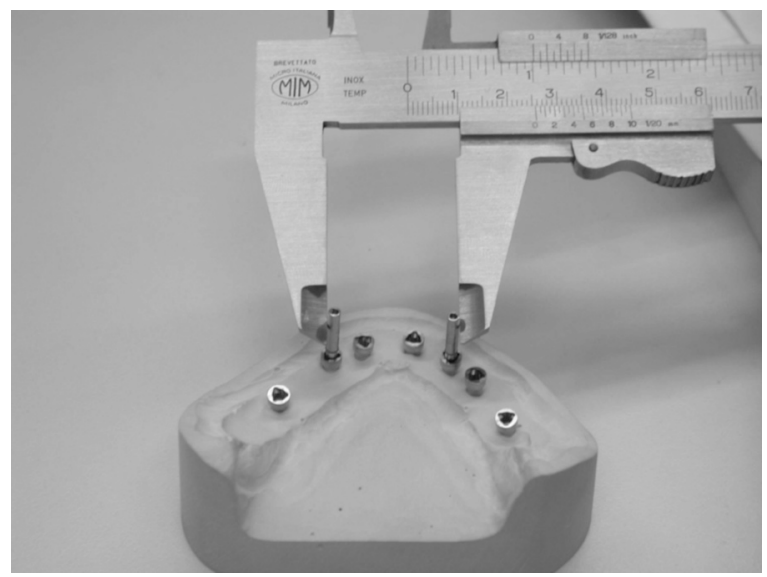

Figure 19: Plaster moulding. 


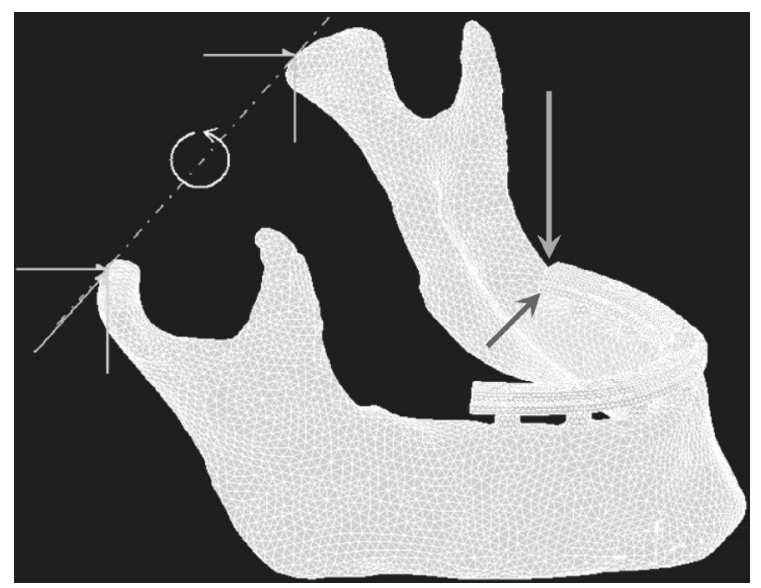

Figure 20: Finite Element mesh.

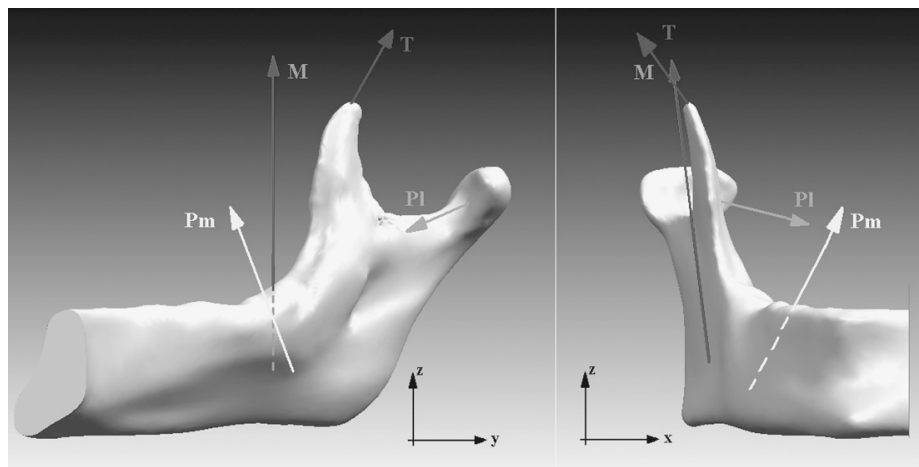

Figure 21: Directions of forces.

Table 4: Directions of forces.

\begin{tabular}{|l|r|r|r|}
\hline & $\operatorname{Cos} \alpha$ & $\operatorname{Cos} \beta$ & $\operatorname{Cos} \gamma$ \\
\hline Masseter & -0.043 & -0.011 & 0.999 \\
\hline Medial pterygoid & 0.587 & -0.165 & 0.792 \\
\hline Lateral pterygoid & 0.714 & -0.692 & -0.106 \\
\hline Temporalis & -0.325 & 0.219 & 0.920 \\
\hline
\end{tabular}

The relationships among the various forces are as follows:

$$
\begin{aligned}
\mathrm{M} & =1.72 \mathrm{Pl} \\
\mathrm{T} & =0.99 \mathrm{Pl} \\
\mathrm{Pm} & =1.15 \mathrm{Pl} \\
\mathrm{Pl} & =\mathrm{F}
\end{aligned}
$$

The directions of the forces may be established by the cosine extracted from geometry considered [21] (Table 4). Figure 21 illustrates the directions of such forces.

WIT Transactions on State of the Art in Science and Engineering, Vol 35, (C) 2008 WIT Press 
Figure 22 displays the stress state due to a vertical load. The most stressed component appears to be the cantilever, where peak stresses occur in the vicinity of the cantilever loaded portion, along its upper face. This fact underlines the need for determining the optimal geometry of the cantilever cross-section, as underlined in Ref. [42].

A critical loading of a multiple replacement is modelled in Figure 20. As the projection of the cantilever beyond the extremal root increases, the stresses significantly increase, although the position of their peak remains unaltered. Figure 22 shows that the maximum stress loci occur in the cantilever in the vicinity of the loaded zone. Although in the case of vertical loading the stress distribution within the multiple replacement depends on the number of roots employed, in the case of lateral loading the stress state is much less sensitive to the number of roots. Figure 23(a) and (b) compare the stress state for 7 (case a) or 5 (case b) roots.

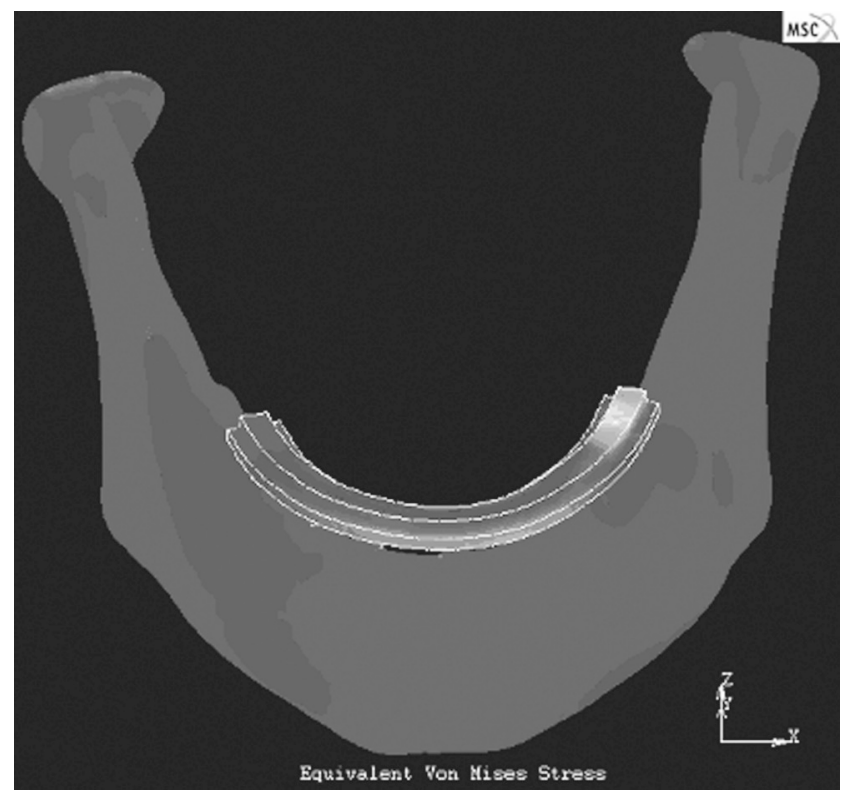

Figure 22: Stresses due to a vertical load.

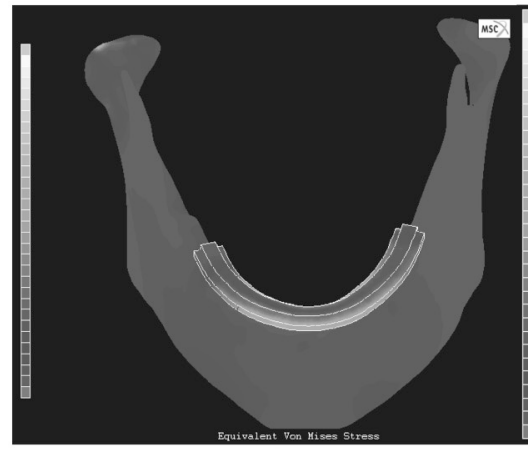

a)

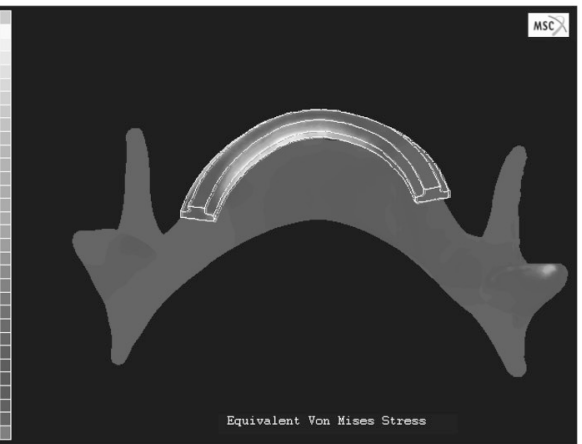

b)

Figure 23: Stress state as a function of the number of roots. 


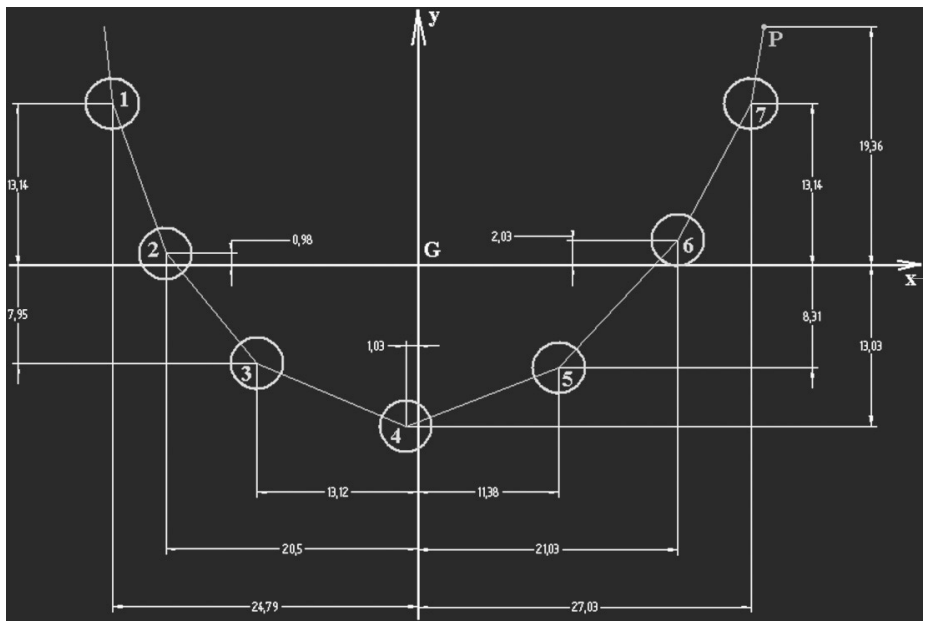

Figure 24: Position of seven roots.

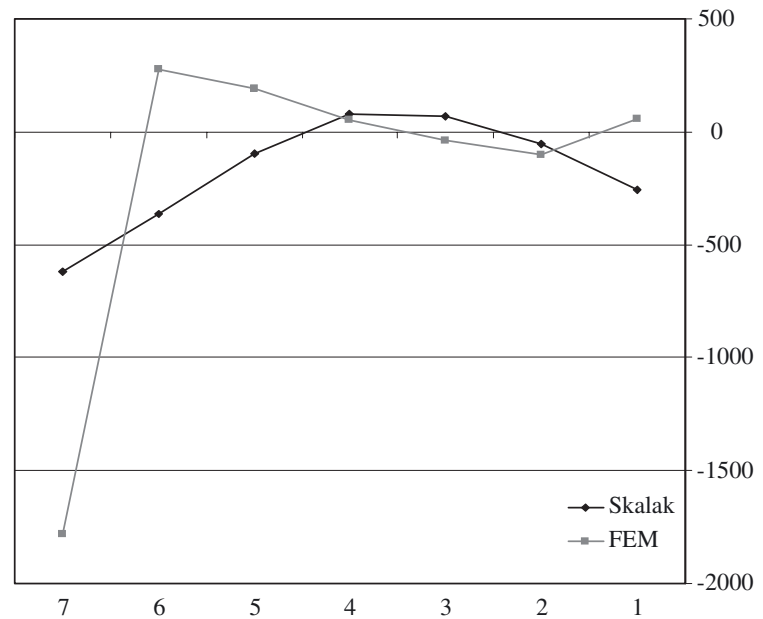

Diagram 1: Vertical loads according to Skalak and to FE.

For a lateral load of $1250 \mathrm{~N}$, (case of bruxism), the percentage of loads borne by the various roots may be computed with finite elements. Figure 24 displays the position of seven roots and of the vertical load. Diagram 1 reports the vertical loads sustained by the seven roots according to Skalak model and the finite elements. A significant difference is evident especially with regard to the most loaded root. The finite element output also clarifies that the load affecting the most loaded root may become even higher than the total load. This is justified by the fact that some loads applied to the roots are tensile.

Two straight (rectified) models have been analysed to justify the previously discussed loadings. In the model (a) of Figure 25 the cantilever is assumed as rigid and it rests on seven deformable roots (Skalak model) as in Figure 16(a), whereas in the model of Figure 25(b) the cantilever is supposed to be flexible whereas the supports are rigid. 


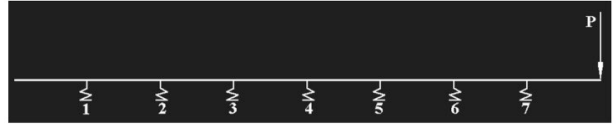

a)

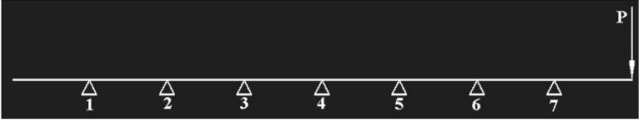

b)

Figure 25: Cantilever model.
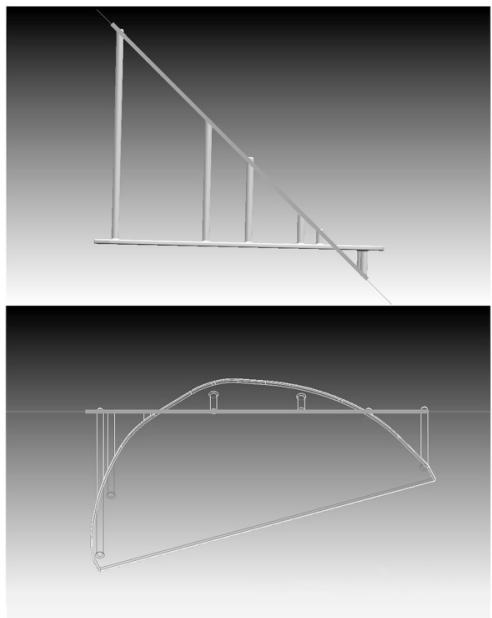

a) Skalak

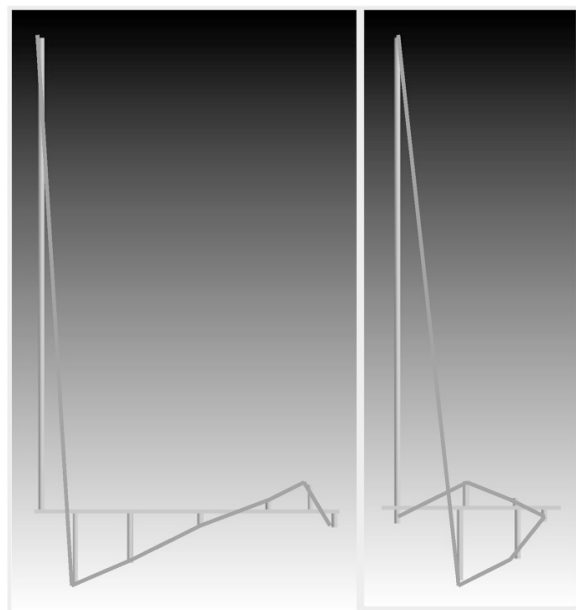

b) FEM

Figure 26: Force distribution of seven roots.

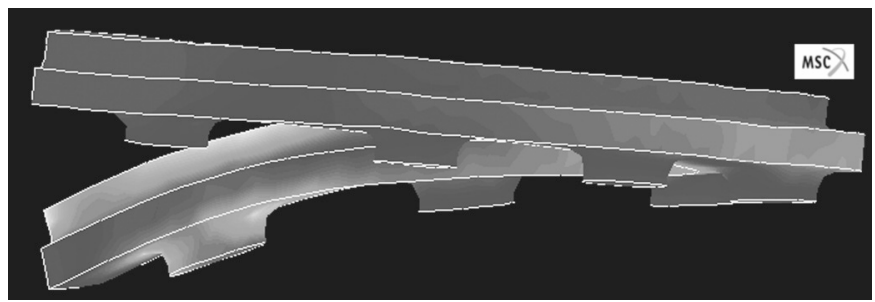

Figure 27: Deformed cantilever model.

Figure 26 presents the force distribution on the seven roots. Figure 26(a) illustrates the Skalak predictions, whereas Figure 26(b) addresses the rigid support forecasts. The two predictions differ deeply.

Figure 27 shows the deformed cantilever in the most realistic, finite element model, in the presence of a vertical load. It is evident that the cantilever zones supported by the roots do no lie on a plane. Finally, Figure 28 compares the finite element predictions with the two approximations of Figure 25. The model based on a rigid support assumption overestimates the highest force, acting on the extreme root, so that it is on a safe side, whereas the Skalak model is on an unsafe side. 


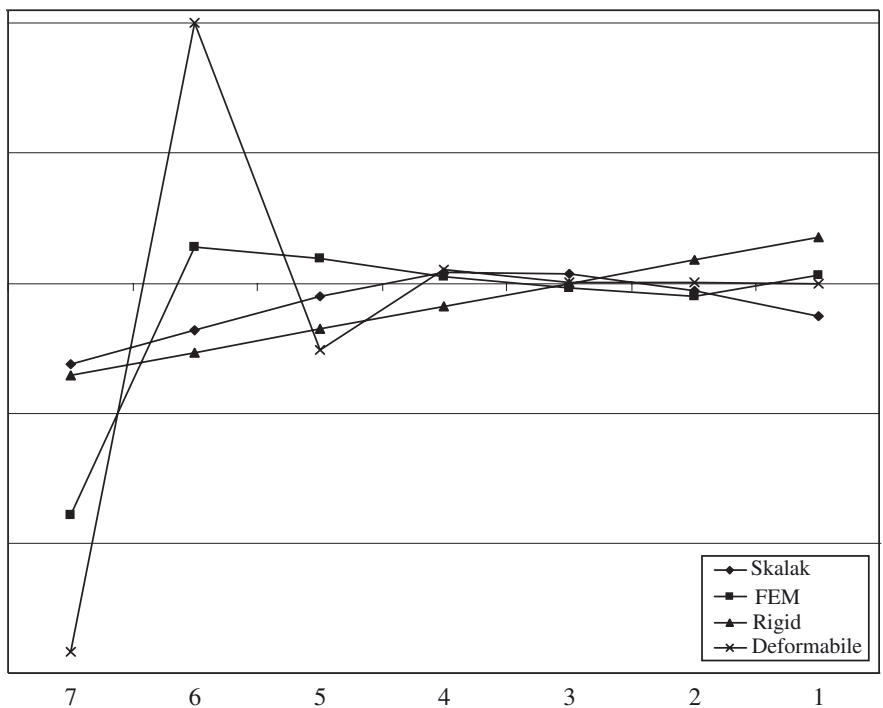

Figure 28: Comparison among various models.

\section{Jaw solid modelling}

The exact knowledge of the jaw geometry is of the utmost relevance especially when dealing with multiple replacements, since in this case the dental implant interacts with several bone regions. The reverse engineering technology appears to be particularly promising in getting a precise jaw external shape, at the cost of missing the internal shape. So, in the case of bones, the reverse engineering approach does not allow the change in thickness of the cortical bone to be measured. This drawback is overcome by the computerised tomography (CT), which allows the bone cortical layer thickness to be retrieved for the various jaw zones.

\subsection{Reverse engineering}

Various commercial reverse engineering technologies are available as optical lasers and laser scanning systems. Figure 29 displays a cadaver mandible, whereas Figure 30 shows its virtual modelling. The principle is exploited of optical triangularisation between the video and the device emitting a laser strip, and not only a single point, on the surface of the object of interest. A mirror oscillating at an angle of $30^{\circ}$ allows a certain surface portion of the object to be covered without moving the laser font. The video captures the image, and the light impulses are then transformed into electrical signals that are digitised and then processed. The precision achievable with the reverse engineering technique based on optical laser is of the order of $0.1 \mathrm{~mm}$. This error is acceptable for most biomechanical applications, apart from contact problems directly involving the scanned bone surface and a biomechanical replacement.

The surface of Figure 31 is defined by a large amount of very small adjacent triangles, hardly perceivable in this figure. A finite element mesh of the whole mandible, including surface and core, could then be generated starting from the above faceted surface mesh. Unfortunately, such approach does not practically allow a mesh refinement possibly requested by the stress analysis requirements. Consequently, a different strategy is usually adopted. With the aid of a 

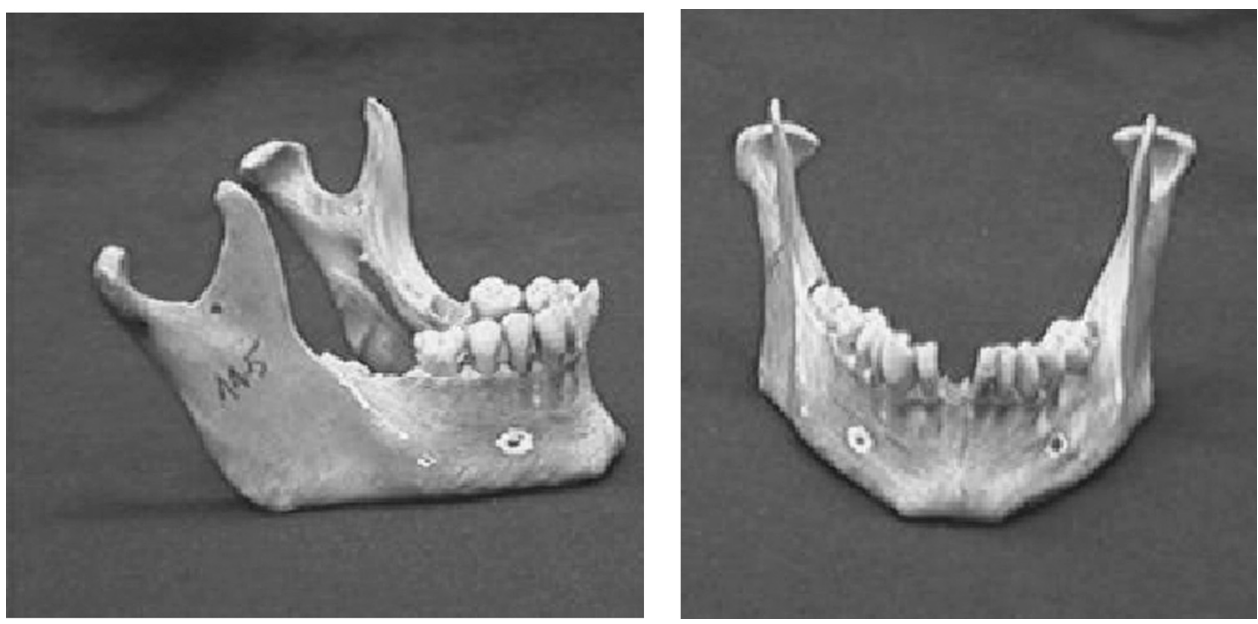

Figure 29: Cadaver mandible.

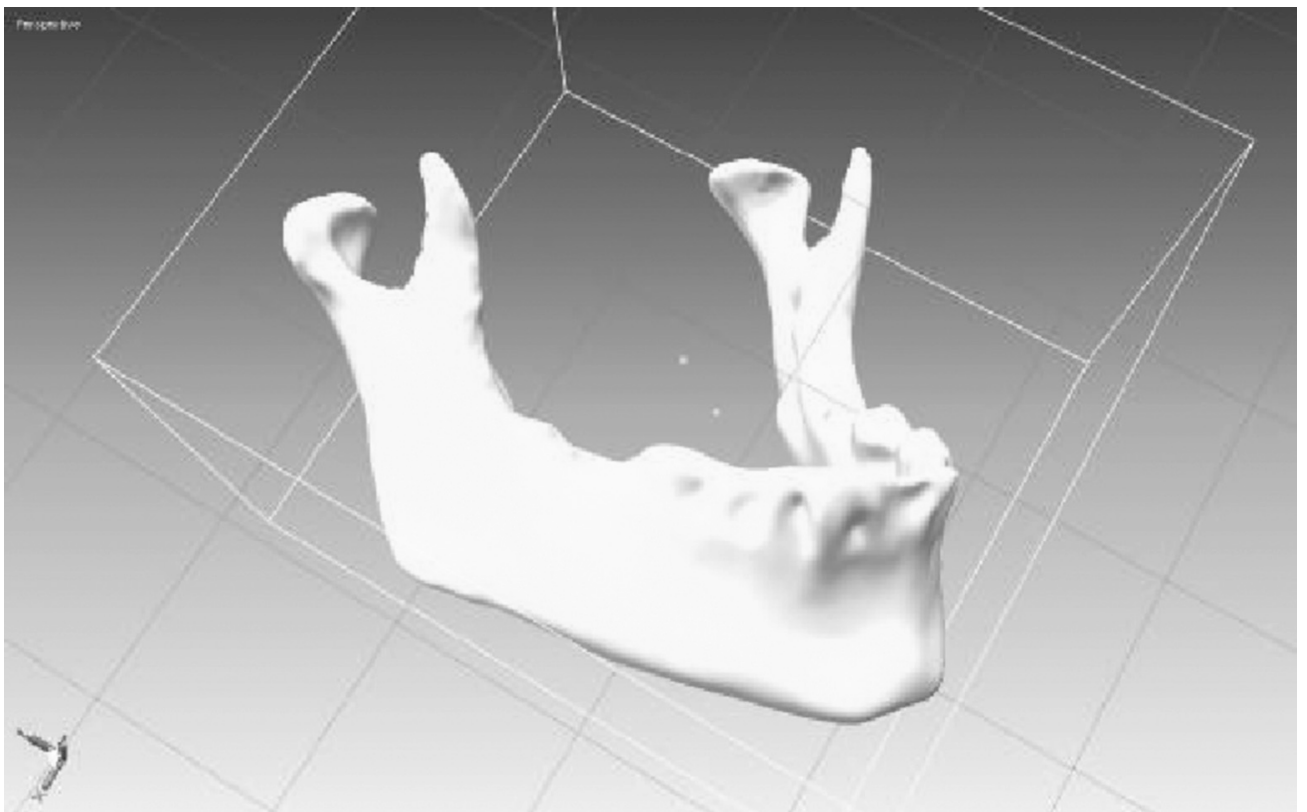

Figure 30: Virtual modelling of a mandible.

dedicated interpolating software, the minutely faceted mandible surface is described in terms of a limited number of contiguous smooth surfaces describing sizeable portions of the original shape. The advance of this smoother geometrical description is to allow increasingly refined meshes to be produced as an input for the finite element stress analysis. For instance, to compare differently sized mandible screwed holes housing the titanium root of the tooth replacement, it is convenient to mesh a mandible whose surface is smoothly defined, rather than starting from 


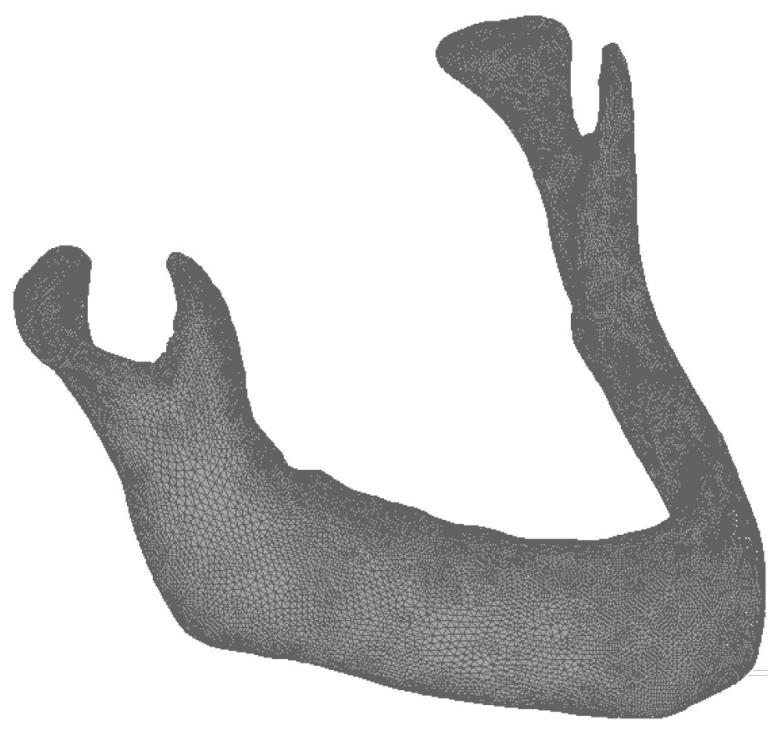

Figure 31: Solid modelling of a mandible.

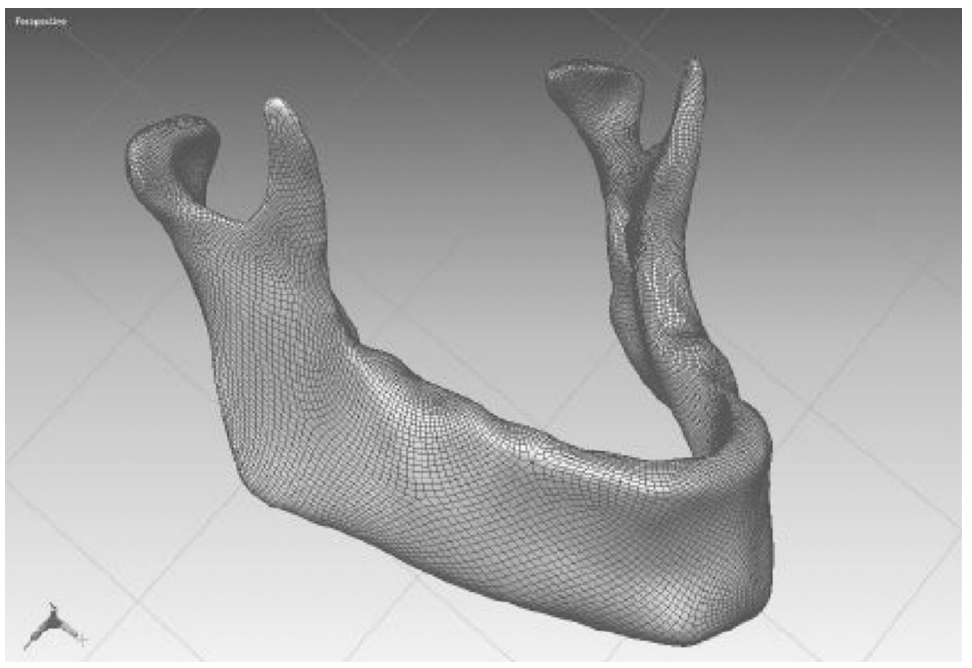

Figure 32: FE mesh.

a triangularly faceted surface. Figure 32 displays the mandible final surface as obtained with a commercial 3D CAD.

A second advantage of a smooth geometrical representation is that it is more easily importable by different softwares. Figures 33 and 34 show the same model imported by various commercial CAD packages.

A1 already underlined, a drawback of the reverse engineering technique is that the thickness of the bone cortical layer cannot be measured. The cortical thickness varies between 1 and $3 \mathrm{~mm}$, so that it cannot be assumed as constant. A manual measurement of the 


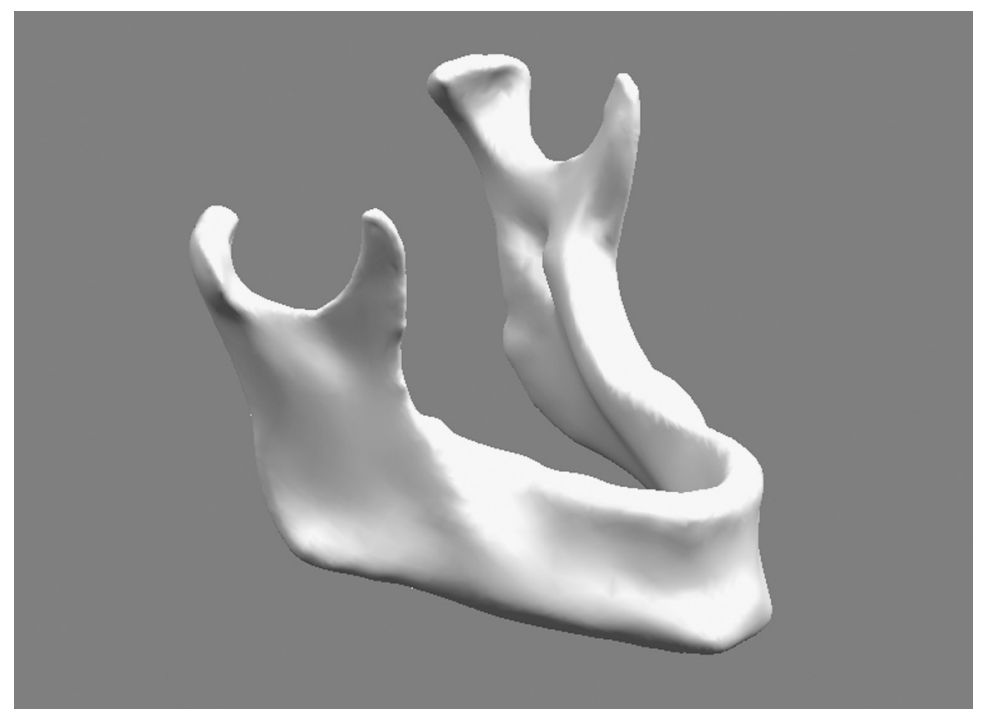

Figure 33: Virtual modelling.

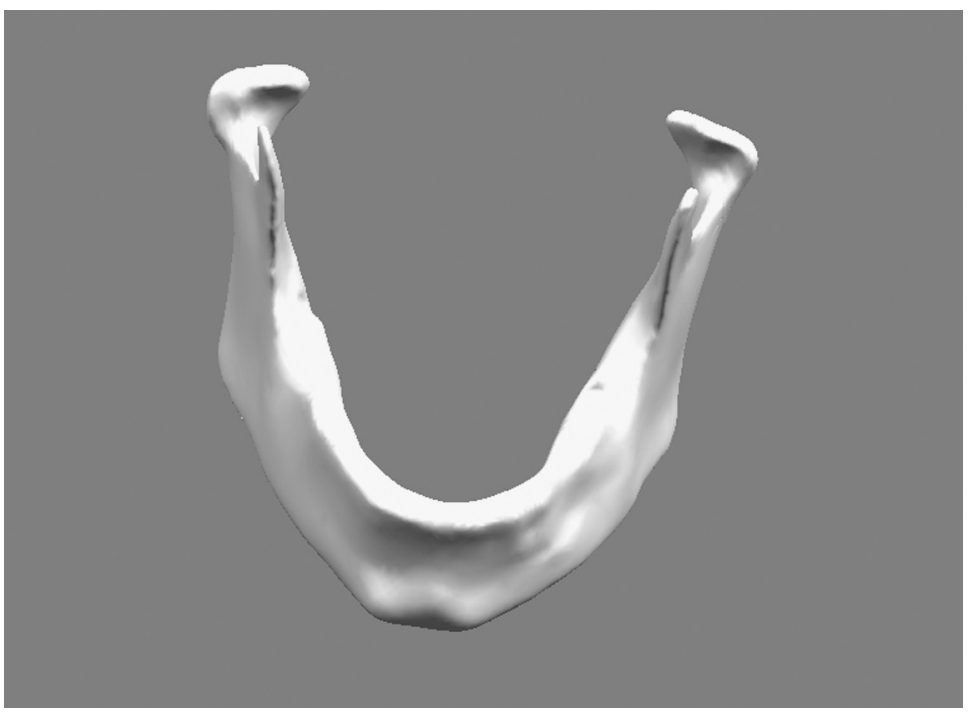

Figure 34: Virtual modelling.

cortical thickness would be feasible in theory but it would be prohibitively time-consuming. The best one can do to supplement the data retrieved with the reverse engineering method is to attribute a constant thickness to macro-regions of the mandible, as proposed by Ref. [39] and shown in Figure 35.

To overcome the problems encountered in defining the cortical layer thickness with the reverse engineering technique, an alternative approach is available, namely the computerised tomography $(\mathrm{CT})$, which will be presented in the next section. 


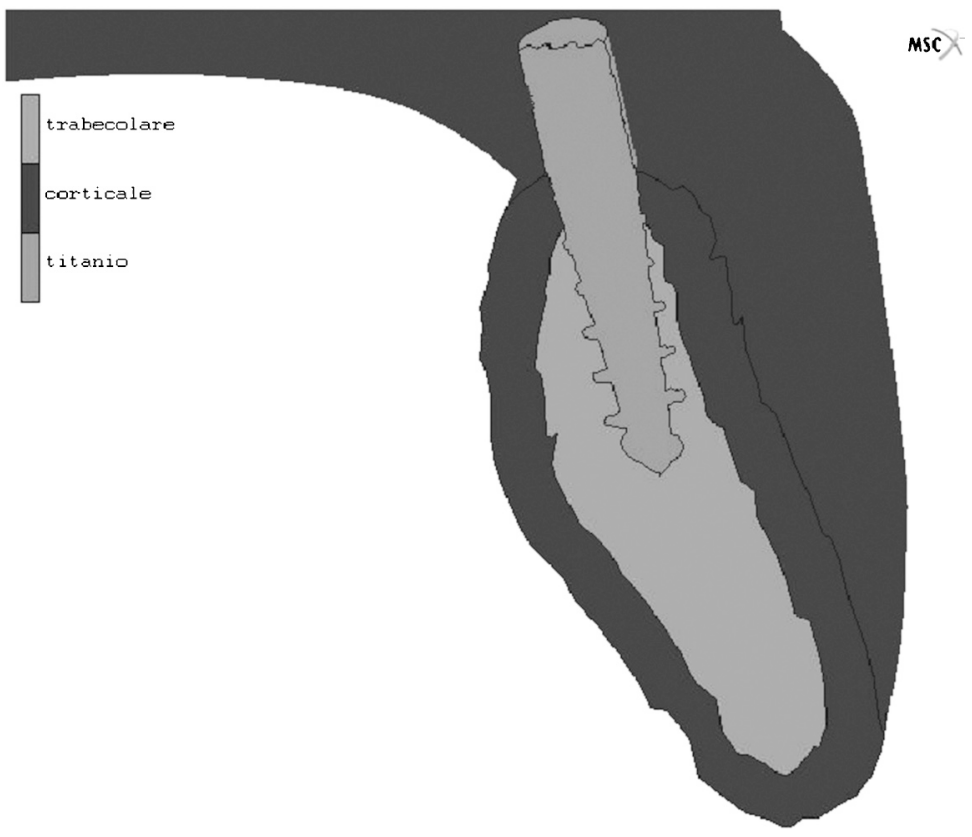

Figure 35: Cortical stratum.

\subsection{Tomography}

With the development of digital imaging techniques, more efficient methods have recently become available for creating anatomically accurate models. These include specialised software for the direct transformation of 2D or 3D information in image data from CT or magnetic resonance imaging (MRI) into finite element meshes. The automated inclusion of some material properties from measured bone density values is also now possible. This approach allows more precise modelling of the geometry of the bone-implant system. In the foreseeable future, the creation of finite element models for individual patients, based on advanced digital techniques, will become possible and perhaps even commonplace.

The $\mathrm{CT}$ is more accurate than a $3 \mathrm{D}$ reconstruction based upon reverse engineering, since it can locally define the actual thickness of the bone cortical layer. In Figure 36 a 3D reconstruction of a human mandible is derived from tomography images of adjacent cross-sections, usually at a distance of $0.75 \mathrm{~mm}$. The different greyscale values visible in Figure 36 evidence the cortical and trabecular zones but, within such zones, they may also be related to the local bone density, thus supplying information on the local Young's modulus. With the aid of specialised software (e.g., Mimics), the adjacent cross-sections are joined to create a 3D model of the whole mandible.

Contrary to a reverse engineering approach, the CT also details the mandible inner portion, and especially the local layer of the cortical zone. It is then possible to give to the output a format compatible with the finite element mesh generators.

Figure 37 compares two mandible meshes, where the left modelling, derived from a reverse engineering analysis, adopts a constant thickness of the cortical bone, whereas the right idealisation, retrieved from a CT approach, accurately mimics the real bone geometry. 


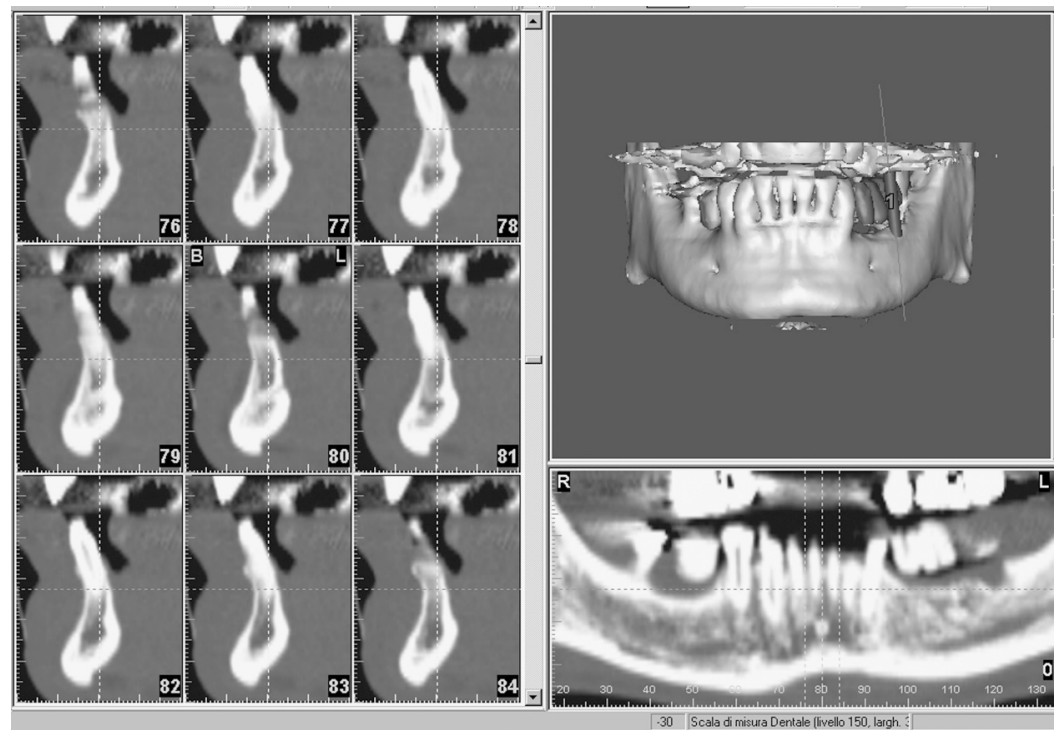

Figure 36: Computed tomography.
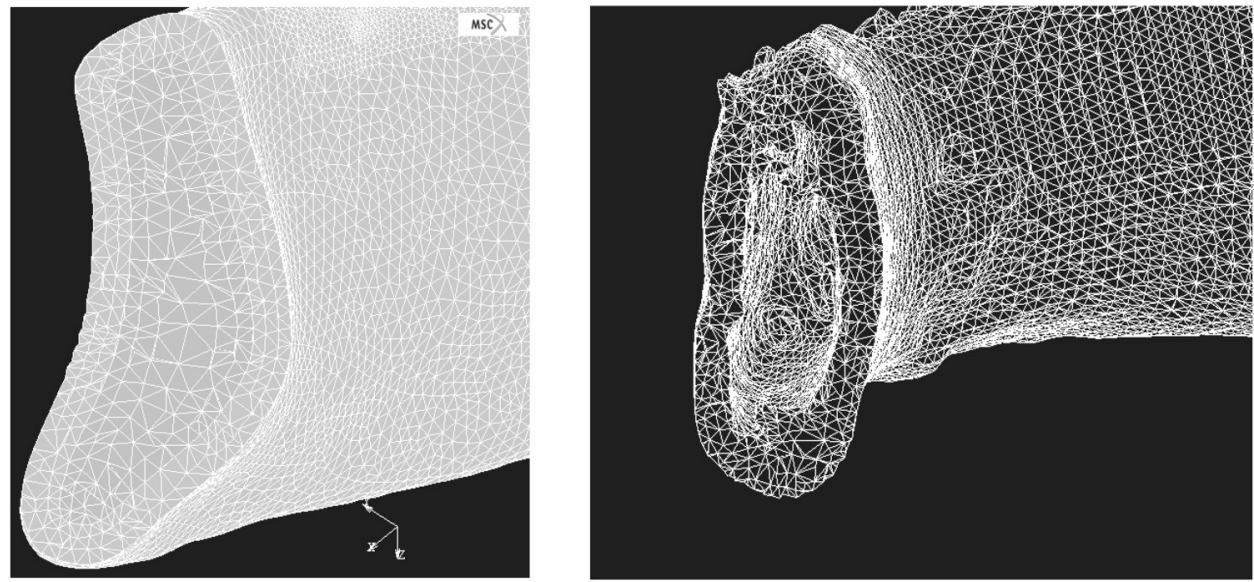

Figure 37: (a) Constant cortical thickness (Reverse Engineering), (b) Variable cortical thickness (TC).

The CT has been frequently employed in 3D finite element stress analysis in dental implants. It has already been noted that the exact knowledge of the mandible geometry is particularly important when dealing with multiple replacements, since in this case the dental implant interacts with several bone regions. It is therefore not surprising that the $\mathrm{CT}$ is mainly employed when modelling multiple replacements.

Among the papers adopting CT in modelling the jaw, significant contributions are by Cruz et al. [21], Jaecques et al. [47] and Geng et al. [48]. 


\section{Various methods of stress analysis}

For a long time, the development of dental fixtures has relied on a trial-and-error procedure. While the importance of field response is paramount, the potential for experimental or numerical testing on models is very high. Fostered by the success experienced in other areas of biomechanics [49], the use of such evaluation techniques has recently been extended also to dentistry for pre-clinical evaluation of implants. Working on models has the advantage to allow a reasonably swift evaluation of parametric changes to signal major effects. This possibility can lead to drastic reductions of cost and time for developing a new design or optimising an existing application.

Among all the methods of analysis available to the biomechanical engineer, two of them have proven especially helpful in implantology: the finite element, boundary element and cell methods, for the computational side, and photoelasticity, for the experimental side. Both techniques are briefly summarised in the following sections. For further details see the excellent contributions by Orr et al. [50] found in the collection edited by Miles et al. [51].

\subsection{Finite element method}

The basic problem of stress or structural analysis according to the finite element method can be succinctly expressed with reference to the structural chain shown in Figure 38. The four parameters involved are force, stress, strain and displacement. They are connected in pairs by three links: equilibrium (involving force and stress), mechanical properties (relating stress and strain) and compatibility (involving strain and displacement). Equilibrium and compatibility are conditions imposed by the laws of mechanics, while for any particular material the nature of the mechanical properties is determined experimentally. In any real structural problem the applied forces and displacements are such that some of each are known ab initio (actions) and the rest are unknown initially (effects). Stress and strain are internal effects and are usually unknown initially. What ensures that a structure (which is stable, i.e. the laws of equilibrium are satisfied, and remains in a compatible state, i.e. the laws of compatibility are satisfied) will behave in a unique manner, which can be solved analytically, is that there always exists a known displacement corresponding to an unknown force and vice versa.

That this is so is true in theory. In practice, things are not so simple. This is because a structure comprises infinity of points on the boundary where forces act and displacements occur and in the interior where stress and strain exist. This is somewhat akin to having an infinite number of simultaneous equations in infinity of unknowns - there may well be a unique solution set, but it cannot be obtained with a finite amount of effort. Stress analysis can be approached from either end of the structural chain depending upon whether the unknown forces or the unknown

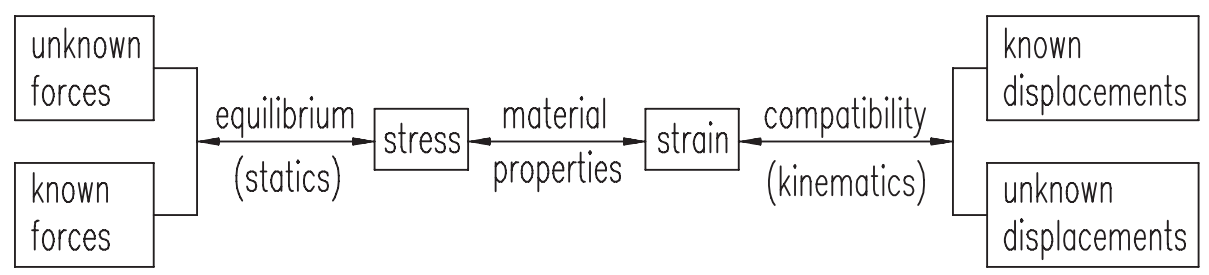

Figure 38: Flow chart of Finite Elements. 
displacements are considered the primary unknowns. If the former is the case, the approach is known as a force (or flexibility) method whereas for the latter the approach is known as the displacement (or stiffness) method. When the force method is adopted the situation is considered as a structure which is effectively statically indeterminate. On the contrary, when approached from the displacement method point of view, the system is considered as a mechanism which is kinematically indeterminate.

Thus any realistic structure is indeterminate to an infinite degree. In order to reduce a problem to a finite degree of indeterminacy so that a solution can be obtained, approximations, idealisations or assumptions, and other simplifications have to be introduced. In the finite element method, the key simplification is to break up the structure into small pieces (finite elements), mutually connected at common vertices (nodes). Within each element, the stress (or displacement) field is established as a known function of the same variable at the nodes. Following this discretisation, the structural problem can be solved in terms of the finite number of unknowns embodied by the master values of stress or displacement competing to the nodes. The quality of the approximation clearly depends on the number of nodes and the order of complexity of the interpolating function over each element.

In recent years, the techniques used to construct these models have dramatically improved due to the development of better classes of elements and strategies for putting them together. The tremendous increase in available computing power and these algorithms have thus made very realistic simulations feasible. The geometry (left) and the finite element mesh (right) of a 2D model of a dental implant are displayed in Figure 39. The principal difficulty in simulating the mechanical behaviour of dental implants is the modelling of human bone tissue and its response to applied mechanical forces. The substantial complexity of the mechanical characterisation of bone and its interaction with implant system has led to major and often incorrect simplifications in many analyses. The principal simplifications usually made in implant modelling are:

1. two-dimensional representation of geometry based on either the assumption of axial symmetry of loads and geometry or the plane strain response;

2. use of static axial loads;
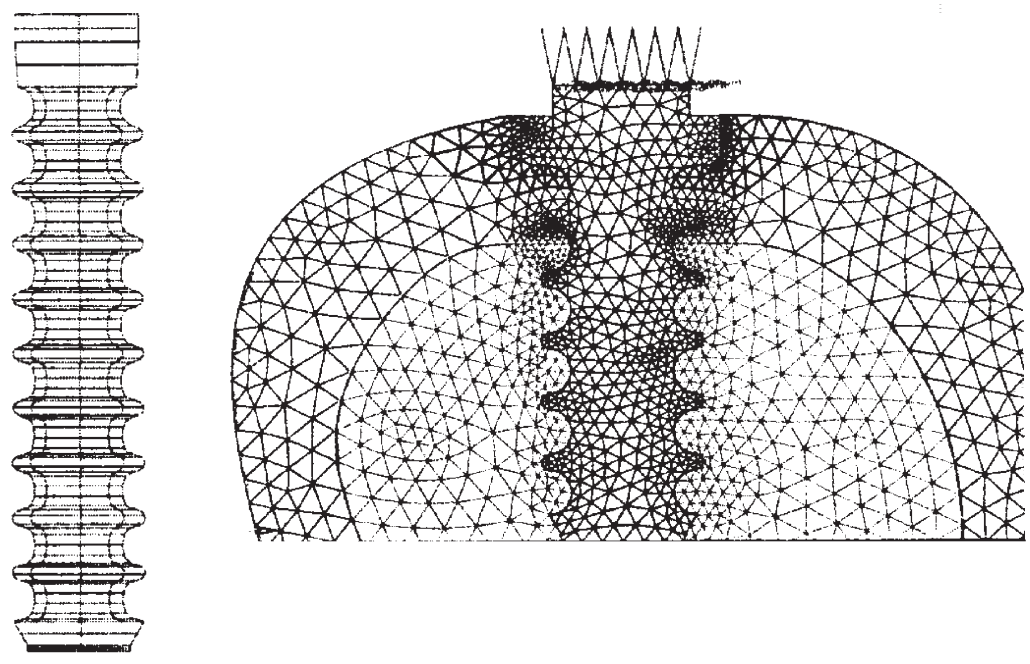

Figure 39: Examples of FE studies. 
3. assumption of homogeneous, linear, elastic material behaviour characterised by a single elastic modulus and Poisson's ratio;

4. assumption of perfect bonding between bone and implant.

Unfortunately, bone-implant systems appear not to completely satisfy any of these assumptions. The geometry and loading are not axially symmetric. A case can be made for assuming plane strain, but this usually leads to the use of artificial boundary conditions that can alter the results significantly. The material is neither homogeneous nor isotropic. Furthermore, the bone appears to need a range of stresses $\left(1.5-5 \mathrm{~N} / \mathrm{mm}^{2}\right)$ for healthy maintenance. Stresses outside this range have been reported to cause resorption of bone tissue. This resorption occurs naturally to obviate high stress concentrations that would ultimately lead to implant failure.

The loads assumed in most analyses to date have been static and directed axially instead of the dynamic cyclic loads directed at the occlusal angle encountered in the jaw during mastication of food. Such loads and their action on the porous cellular structure of trabecular bone have not been subject to much study. The contact between bone and implant (osseointegration) is often modelled as a perfect bond - a situation that exists in only a few cases.

Many of these limitations have been overcome by the recent finite element study by Patra et al. [51]. Models have been constructed on the mechanical behaviour of an embedded dental implant and the surrounding bone where resorption is known to occur. Semi-realistic (2D, Figure 39) and realistic (3D, Figure 40) models were created of the human mandible and of several implant geometries including conventional and alternate designs. Figure 40 shows the mandible interpreted as a 3D bone (left) and its 3D finite element idealisation (right).

From the results of the analysis performed it was seen that the cortical bone was carrying most of the load. It was also observed that stresses in the nearby trabecular region were significantly lower, sometimes by ten times or more. This pattern of stress behaviour was typical to all implant types analyzed.
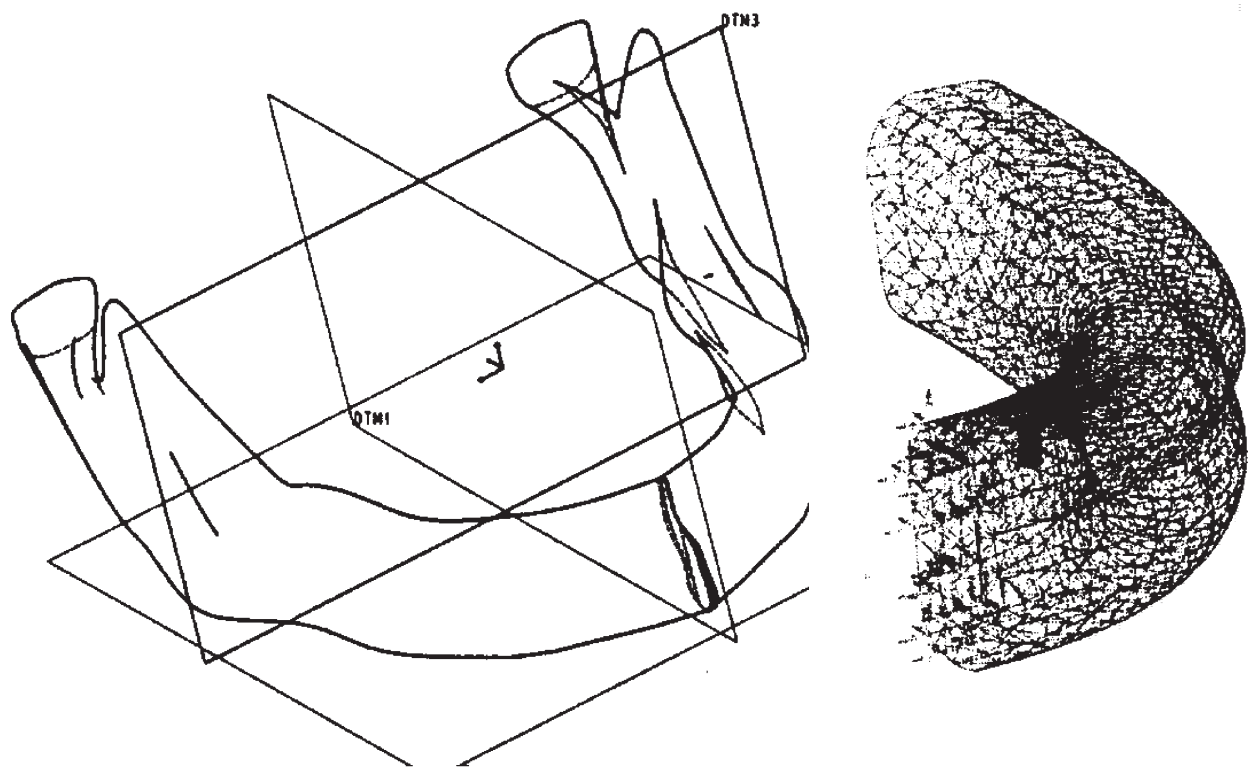

Figure 40: Three dimensional model and mesh of a mandible. 
It was also observed that in the 3D analysis, there is little, if any, stress in the trabecular tissue at the apex of the implant. The artificial constraints used in the 2D analyses cause a false high-stress in this region as several previous papers would indicate. Replacement of the constraint boundary condition by a spring with stiffness derived from cadaveric tendon data yields much better results. The results are also affected by progressive bone loss, anisotropy and fatigue effects.

On comparing the stress levels of both the BUD and Brånemark implants, the above study found that the latter seemed to exhibit several heightened stress levels in bone that were absent in the former. This was primarily due to the different thread profiles and differing shoulder contours. The BUD thread profile seemed to distribute stresses more evenly through the trabecular tissue. There also seemed to be significantly fewer stress risers in the BUD implant in comparison with the Brånemark implant. Furthermore, maximum stresses were significantly lower in the BUD design under the same loading conditions.

It has already been noted that in most studies the bone is locally assumed as homogeneous, where its trabecular architecture is not mimicked in detail. In some studies a modelling of the bone microstructure has been attempted, but such approach requires massive computer resources. For instance, in Van Rietbergen et al. [52] a modelling of $1 \mathrm{~cm}^{3}$ of trabecular bone involved more than one million degrees of freedom.

\subsection{Boundary element method}

The boundary element method constitutes an alternative to the finite element approach. The most important practical feature of the boundary element method is that it requires only a discretisation of the component surface rather than of its volume, thus considerably reducing the mesh generation time, the degrees of freedom involved and, consequently, the numerical solution time with respect to a finite element meshing. Also a mesh improvement suggested by the trend of the stress gradients obtained with the initial solution is eased with respect to a fully $3 \mathrm{D}$ meshing. The boundary element method is particularly advantageous over finite elements when only the surface displacements and stresses are required, as in the evaluation of stress concentrations or in unilateral contact problems, e.g., in biomechanical implants. On the contrary, the boundary element method loses most of its appeal when non-linear elasticity is involved, e.g., when large deformations and displacements are encountered, or when an elasto-plastic modelling is required.

Most of the advantages of the boundary element method stem from its complex mathematical foundations. A key point is the employment of the Green theorem, according to which a 3D problem governed by equations valid within the body volume may be reformulated as a 2D problem ruled by equations valid over the body surface, thus reducing by one the problem dimension. A distributed pressure acting along the body border is interpreted as a sequence of infinite, adjacent, infinitesimal, concentrated loads. This interpretation underlines the importance of knowing the stress distribution due to a single point force, named the fundamental solution. If the pressure is constant along a border segment, the stress due to such pressure distribution may be derived from the stress activated by a point load through analytical integration of singular integrals. For less simple pressure distributions, this integration may not be performed analytically, which in turn requires a problematic numerical integration of awkward singular integrals. A recent paper illustrating how to reformulate the problem in order to avoid the appearance of singular integrals has been presented by Liu et al. [53].

Moving to studies specifically addressing bone modelling, a comparison between 2D and 3D finite element and boundary element forecasts has been presented in Ref. [54] with respect 
to a titanium dental implant in the surrounding of the mandible bone, where a simplified bone mechanical characterisation has been adopted. In addition, a boundary element stress analysis of a mandible has been carried out in Ref. [55]. The bone inhomogeneity and anisotropy have been taken into account. The main scope of this study was to examine the loads acting on thecondole during mastication.

\subsection{Cell method}

The numerical approach named cell method has recently been introduced by Tonti [56, 57] and, among various applications, it has been proposed to model porous materials such as sintered alloys [58] and bones [59]. Since the cell method is not based upon a differential apparatus to formulate the equilibrium equations, it is claimed to be more suitable than the finite element approach when modelling materials where 'the size of the heterogeneities is the same scale of that of the discretisation' [60]. According to [56], the variables ruling an elastic problem may be classified into two main groups; the configuration variables and the source variables. (A third group encompassing energy variables is not considered here.) The configuration variables include the displacement and the strain tensor, whereas the source variables are the stress tensor and the forces and moments.

One of the fundamental aspects of the cell method is that two distinct meshes are associated to the two sets of variables. If a triangular mesh is used to treat the first group of variables, where the nodes fall at the vertices of the triangles, the dual mesh is composed by contiguous cells formed by polyhedrons surrounding the various nodes. More precisely, the periphery of the cells is defined by consecutive segments connecting the centres of mass of the various triangles surrounding a single node. In Figure 41 a very restricted triangular mesh is illustrated together with the dual cell, shaded in the interest of clarity. The dual cell may be regarded as the influence region for the node internal to the cell itself. In fact, the equilibrium equation is formulated in a discrete fashion for each dual cell, that is, for the influence region of each node; it simply expresses the equilibrium of the forces transmitted along the whole boundary of each dual cell, by computing the stresses for all triangles surrounding the node of interest. With the cell approach there is no need to resort to energy concepts or to rely on continuity to formulate the equilibrium equations. The result of this approach is a linear system of equations of the kind:

$$
\{F\}=[K]\{\delta\}
$$

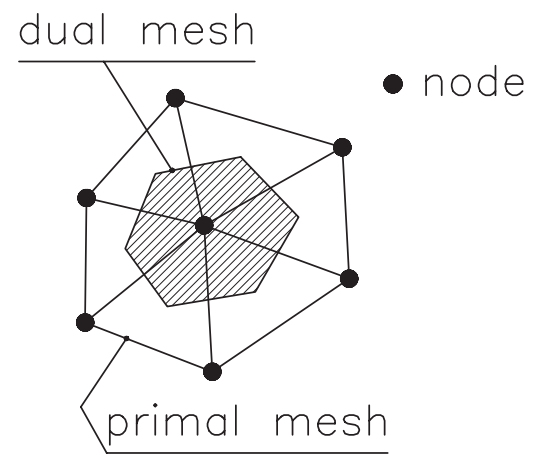

Figure 41: Primal and dual cells. 
where, as for the finite element approach, $\{F\}$ is the vector of the external forces, $[K]$ represents the stiffness matrix, and $\{\delta\}$ is the displacement vector.

The example of Figure 41 is based upon a triangular primal mesh, that is, to a linear interpolation of the displacement field, which in turn implies constant stresses within each triangle. In this case the stiffness matrix coincides with that computed according to the finite element method, whereas the known variable vector is different [57]. Better results with respect to the finite element approach are achieved by considering higher-order interpolation functions. For example, for a six-noded triangular mesh, the stiffness matrix no longer coincides with the mesh generated by the finite element approach [57], and the convergence rate has been shown to be comparable to or better than the finite element forecasts.

Moving to studies specifically addressing bone modelling, in Ref. [59] a digital radiography image of the bone cancellous zone was performed as an input for a plane mesh including up to 19487 nodes, aimed at mimicking the effects of osteoporosis on the bone bearing capabilities.

In [58] plane and preliminary 3D models of a cancellous geometry were modelled with the cell method, where the results obtained seem encouraging.

It may be concluded that, although applications of the cell method to complete biomechanical problems have not been developed yet, the cell method seems a promising approach and a valid alternative to more consolidated numerical methods.

\subsection{Photoelastic modelling}

The photoelastic technique involves the observation of patterns of dark and light lines, known as fringes, which result from interference of components of plane polarised light when transmitted by a stressed plastic model. The experimental examination of photoelastic models is carried out in an instrument known as a polariscope, the main optical elements of which are detailed in Figure 42. When plane polarised light is transmitted by a stressed plastic model two

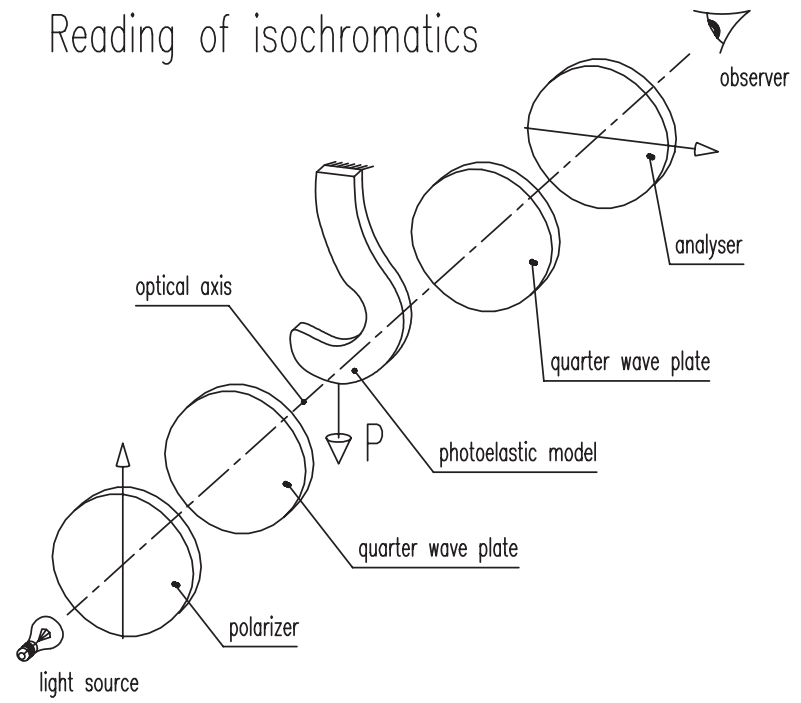

Figure 42: Polariscope. 
groups of dark fringes are visible, isoclinics and isochromatics, which give information about directions and magnitudes of principal stresses, respectively. Each of the main optical elements, called the polariser and analyser, only transmit light along one axis. Thus the plane polarised light which is incident on a model may be considered as a transverse wave lying in a single plane. The polariser and analyser are normally set with their axes at $90^{\circ}$ to each other.

When a 2D plastic model is stressed in the loading frame, the material becomes 'double refracting'. This means that light is transmitted along two perpendicular axes which are aligned in the direction of the principal stresses at any point in the plane of the model. If one of these principal stress directions coincides with the plane of the incident polarised light then the light will be transmitted through the model but will then suffer extinction when it encounters the analyser set out at $90^{\circ}$ to the initial plane of polarisation. Hence dark fringes (isoclinics) will result along the loci of all principal stresses whose directions coincide with the polariser/ analyser axes. Other areas of the model will appear light. Rotation of the polariser and analyser, while maintaining the perpendicular relationship of their axes of transmission, allows the loci of principal stresses in all directions to be identified. The two light waves transmitted at any point of the stressed model not only coincide with the directions of the principal stresses but are transmitted with velocities that depend on the stress magnitudes. The transmitted waves will have varying phase relationships as they exit the model, which allows constructive and destructive interference and hence the dark and light isochromatic fringes. This interference is of course wavelength dependent, so fringes are only clearly defined dark and light regions if a monochromatic light source is used. The resulting fringes are loci of constant principal stress difference, or constant shear stress, and are identified by number as they appear sequentially with increasing model loads. They may be related to actual stresses by a simple formula.

The quarter wave plates illustrated in Figure 42 are used to permit viewing of isochromatics without the isoclinics being superimposed.

Photoelastic methods have been applied to biomechanical studies since the late 1930s, particularly to investigate stresses in bone and implants in the orthopaedic [50] and dental fields. The ready indication of principal stress directions has made photoelasticity particularly suitable for study of the directional properties of cancellous bone and its response to placement of implants [61]. The applications of photoelastic methods to model dental structures reflect developments in restorative dentistry since 1949. Early publications generally relate to cavity restorations in teeth $[62,63]$. Scrutiny of stresses in designs of removable partial dentures is followed with later analysis of fixed bridges $[64,65]$.

Only in the last decade, photoelasticity has been used to contribute to the development of dental implants where direct fixation of prostheses is made to the bone of the jaw [66]. In the case of dental implants, usually the actual implant is inserted into the photoelastic material and loaded to produce fringes around it. An isochromatic pattern obtained in this way is shown in Figure 43. The fundamental information supplied by such a picture is the regularity of the stress distribution along the body of the implant. Detection of hot spots where exceeding stress concentrations can be harmful to the bone is readily made by simple observation of the fringe accumulation in the embedding resin. A major difficulty in studying implant-bone interaction by photoelasticity is the correct reproduction of the bone modulus of elasticity, which greatly affects the stress distribution. A guideline for identifying the photoelastic materials that correctly matches the biomechanical context is presented by Little [67] and Little et al. [68]. Photoelastic studies in which an attempt is made to mimic bone cortical and cancellous zones with two materials reproducing the two different Young's moduli are rarely met, due to the practical problems encountered in making the models. A distinguished example of a photoelastic study adopting two different materials for cortical and cancellous zones is [69], where the human 


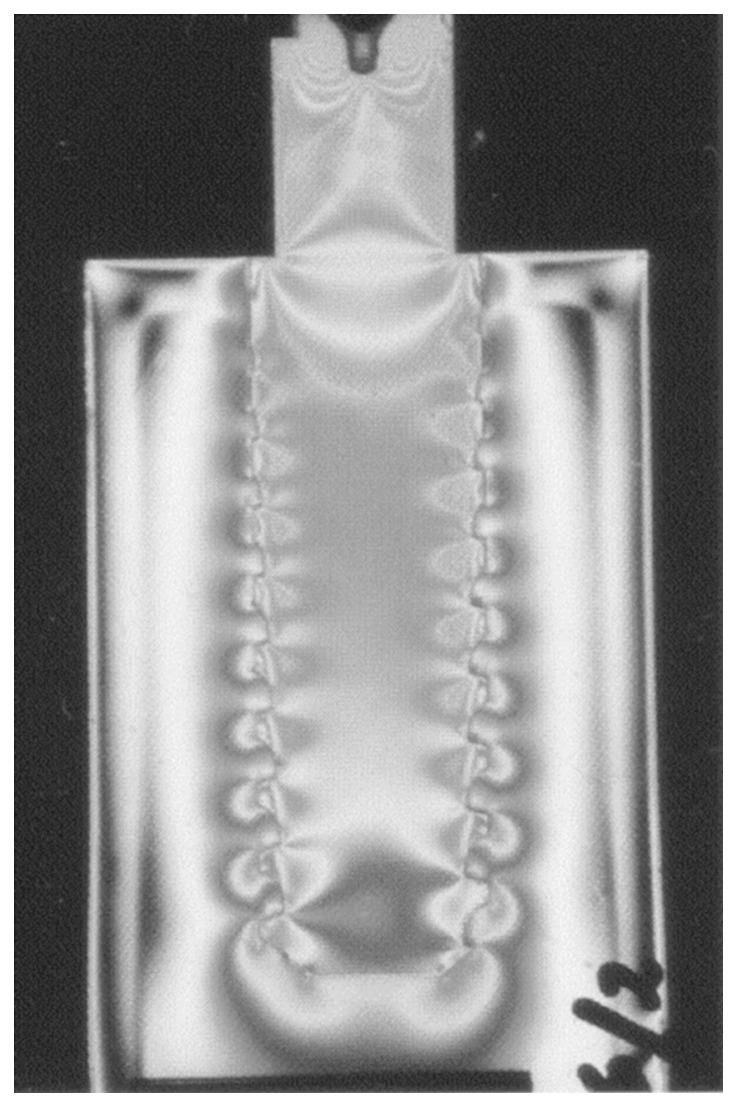

Figure 43: Photoelastic fringes.

knee joint contact pressure distribution is addressed. Papers employing photoelasticity to deal with dental prostheses are Refs. [70-72].

\section{Stress analysis results}

This section aims at showing the potentials of a stress analysis addressing a dental implant. As it is evident from the previous sections, it is not yet practically possible to mimic some complex features of the bone and of the bone-implant interaction. As a consequence, the limits of the stress analysis currently feasible will be underlined throughout the presentation of the results.

The stress analysis of a single dental implant addresses two distinct, relevant zones, namely the bone-implant root interaction, and the contact problems among the various metal components of the tooth replacement. Concerning the first zone, one of the main goals of a stress analysis of the bone-root implant interaction is to design the implant root so that the stresses within the bone by the contact zones be evenly distributed [73]. Screw pitch, thread profile and length, implant diameter and angle of inclination of the implant, and profile of the root zone contacting the cortical layer are among the most investigated aspects. The body of the scientific literature 
signals that the cortical bone-implant interface exhibits a high stress concentration [74]. In general the allowed stress within the cortical bone is lower than, say, $5 \mathrm{MPa}$, in order to limit bone resorption. In Ref. [28] it is found that a threshold of $30 \mathrm{MPa}$ produces a new equilibrium geometry, that is, appreciable bone resorption.

Some simplifying assumptions are usually introduced to make the stress analysis of the bone root interaction more tractable. The cortical and cancellous zones are modelled by using different Young's moduli and Poisson's ratios, but the bone inhomogeneity and anisotropy are usually ignored. Some pioneering studies are available which model the trabecular structure at a microscopic scale, but these numerically prohibitively demanding models cannot yet be employed in the analysis of a whole biomechanical implant.

Full osseointegration between implant root and bone is generally assumed, [75], although loss of osseointegration has often been signalled, [76].

The physiological values of the mastication load are of the order of $100 \mathrm{~N}$ for the axial force and of $20 \mathrm{~N}$ for the lateral force [28], but the load can reach values as high as $500 \mathrm{~N}$ (Menicucci et al., 2001), and it is usually assumed to act obliquely with an angle from the vertical axis ranging between $0^{\circ}$ and $30^{\circ}$ [77-79] and often up to $45^{\circ}$.

The single dental implant interacts with a somewhat limited bone portion, so that simplified 2D models of the bone structure are often assumed as an alternative to fully 3D approaches, especially when the root shape has to be optimised through a massive number of numerical simulations. Among the 2D models, axisymmetric and plane idealisations have been proposed $[27,56,73,80]$.

If the problem is modelled as plane, the effects of an oblique loading may be mimicked, whereas if the problem is assumed as fully axisymmetric, the load can only act vertically. Since the effects of the load inclination are deemed to be relevant, an intermediate idealisation between a fully $3 \mathrm{D}$ and an axisymmetric modelling is achieved by adopting an axisymmetric mesh, but loaded by an oblique force. So doing, a simplification of the meshing labour is achieved, but the computing time remains that of a 3D study.

Going back to the plane models, since the tooth under scrutiny is (hopefully) surrounded by two teeth, the load transverse component is particularly detrimental when acting along the medio-lateral (bucco-lingual) plane [81] (Figure 44). As a consequence, the best plane model appears to be that mimicking the mandible cross-section.

Figure 44 shows a typical mesh of the titanium root and of the surrounding bone portion, where the mesh becomes particularly dense at the root-bone interaction.

Figure 45 illustrates two plane models, the left one based upon the medio-lateral (buccolingual) cross-section, and the right one adopting a mandible section perpendicular to the previous one (sagittal plane). The titanium root and the bone cortical and cancellous zones are clearly perceivable.

Figure 46 presents a typical finite element output showing the location of the stress concentrations within the bone, and Figure 47 shows an enlargement of the cortical bone zone affected by high stress levels. For the sake of clarity, the root has not been shown. Figure 48 illustrates the stress response to a modification of the neck zone, signalling an undesired localised highly stressed zone at the cortex upper side. Finally, Figure 49 addresses the same zone of the previous figure, and it illustrates the results of an optimisation study aimed at evenly distributing the stresses at the cortical zone-implant interface. The best shape for the root neck appears to be a spherical geometry [82], which unfortunately contrasts with the practical implant possibilities.

Moving to the stress analysis concerning the contact problems among the various metal components of the tooth replacement. 


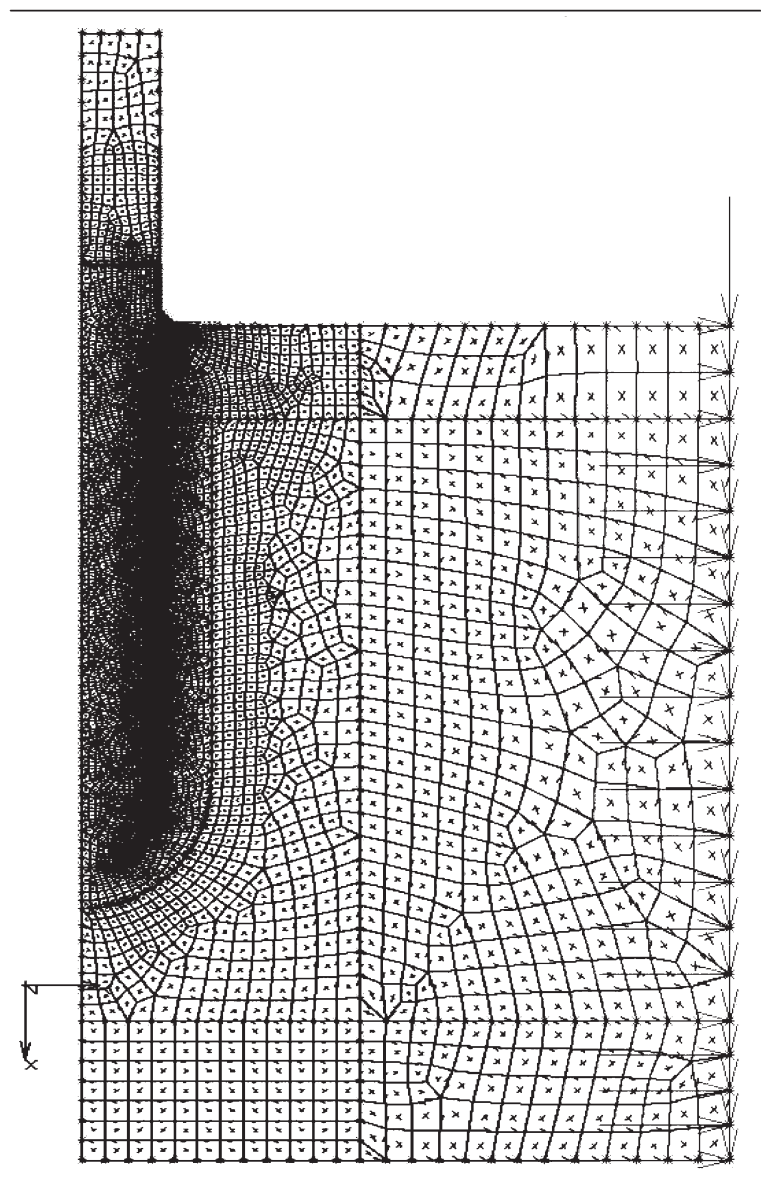

Figure 44: FE plane model.
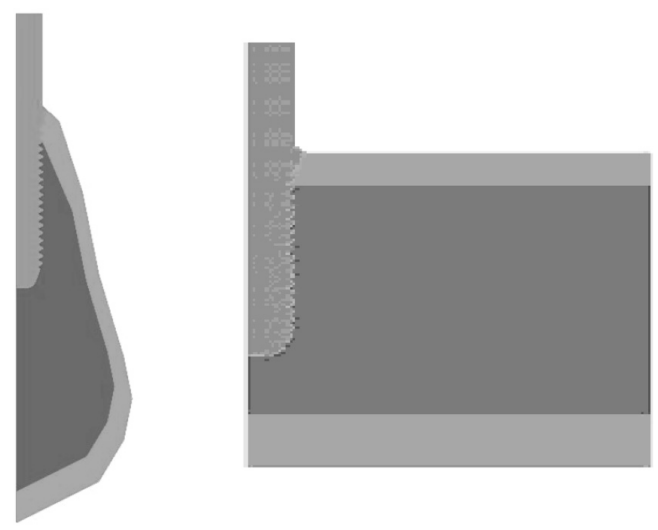

Figure 45: Two plane models.

WIT Transactions on State of the Art in Science and Engineering, Vol 35, (C) 2008 WIT Press www.witpress.com, ISSN 1755-8336 (on-line) 


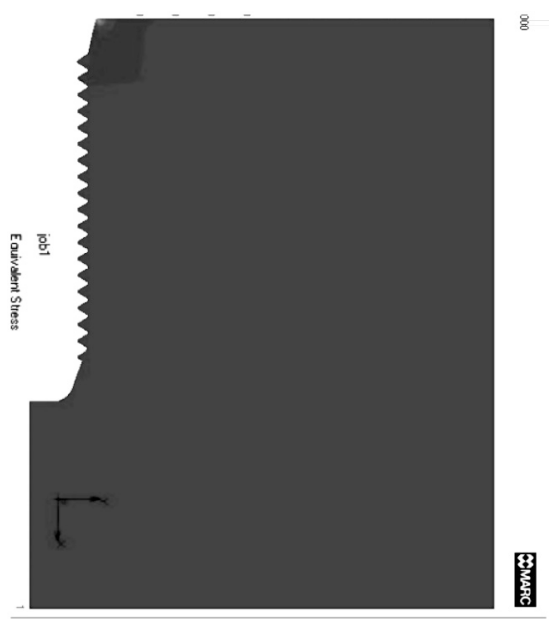

Figure 46: FE output.

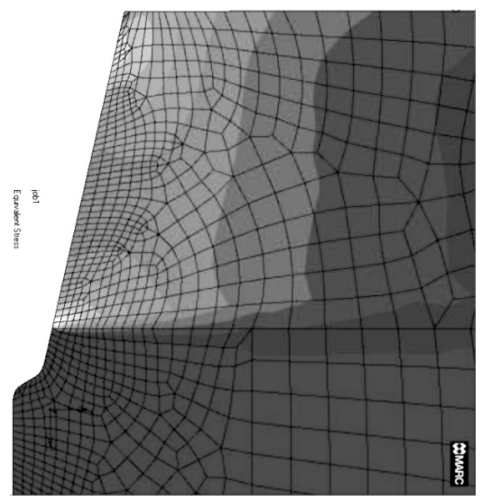

Figure 47: Enlargement of Figure 46.

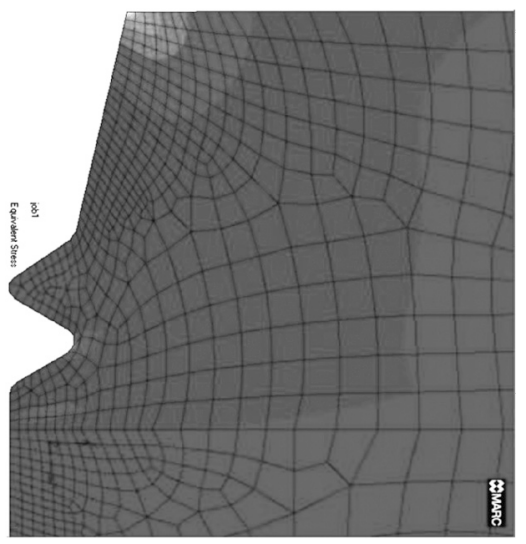

Figure 48: Stress concentrations.

WIT Transactions on State of the Art in Science and Engineering, Vol 35, (C) 2008 WIT Press www.witpress.com, ISSN 1755-8336 (on-line) 


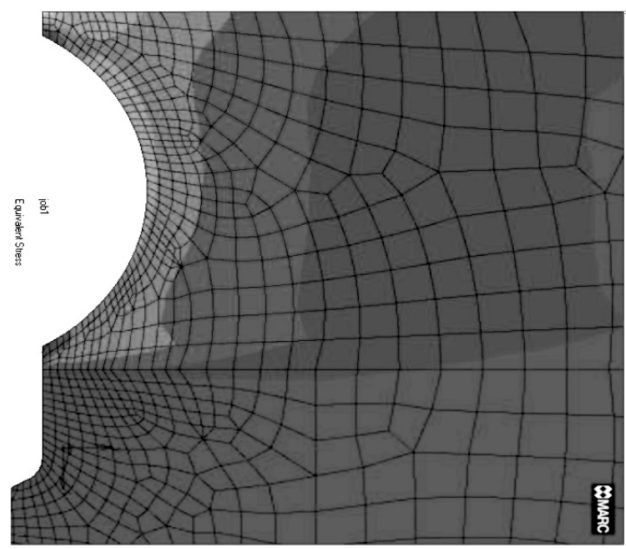

Figure 49: Stress concentrations.

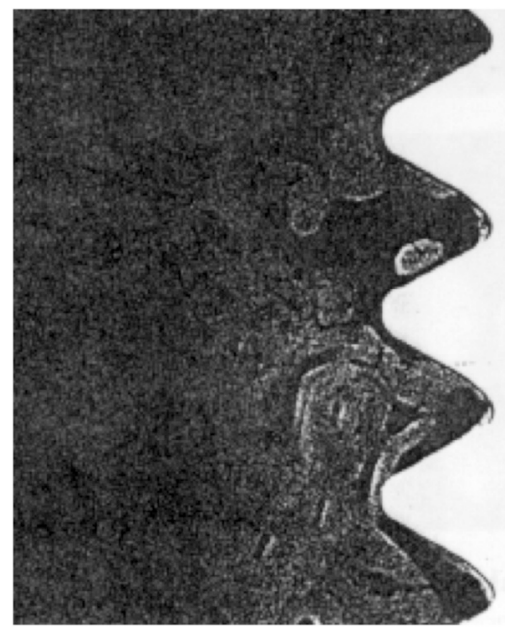

Figure 50: Properly remodelled bone layer.

\section{Biological aspects}

The long-term biomechanical success of an endosseous implant is primarily determined by the degree of stability of the fixture (pillar) surrounded by the bone. Two principal causes contribute to excessive mobility and early failure. The first cause, which especially affects the osseointegrated devices, is the poor immobilisation of the implant after surgery. This type of failure arises either from the negative biological response of the bone to the implant material (low biocompatibility) or from the premature loading of the implant (direct mastication or indirect jaw deformation) in the post-surgical rest period. An essential condition for bone remodelling around the fixture is the absence of any loads for a period of 6-20 weeks after surgery. The second cause of failure is ascribable to the excessive mechanical stress experienced by the bone enclosing the implant. A micro-radiography of the properly remodelled bone layer (left) around the implant (right) is shown in Figure 50, demonstrating a good bone formation 
to the threaded geometry of the fixture. High stress levels, due to abnormal loading conditions on the restored tooth or improper choice of implant size, result in gross resorption of the newly formed bone (Figure 51) and loosening of the prosthesis. In the aftermath of bone resorption, fatigue fracture of the implant (Figure 52) is often to be expected.

Structural failure of the implant can also occur in the absence of bone resorption. It is chiefly traced to under-dimensioning of the implant (low fixture diameter), oversizing of the prosthetic

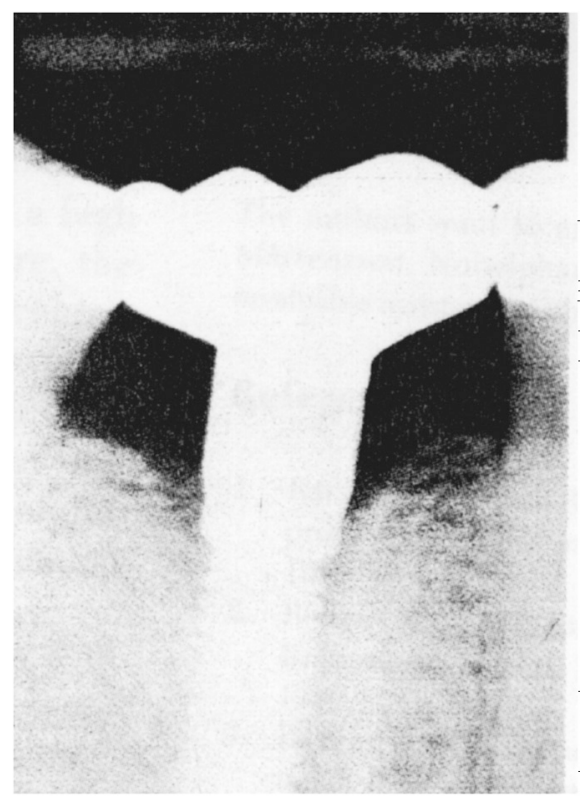

Figure 51: Bone resorption.

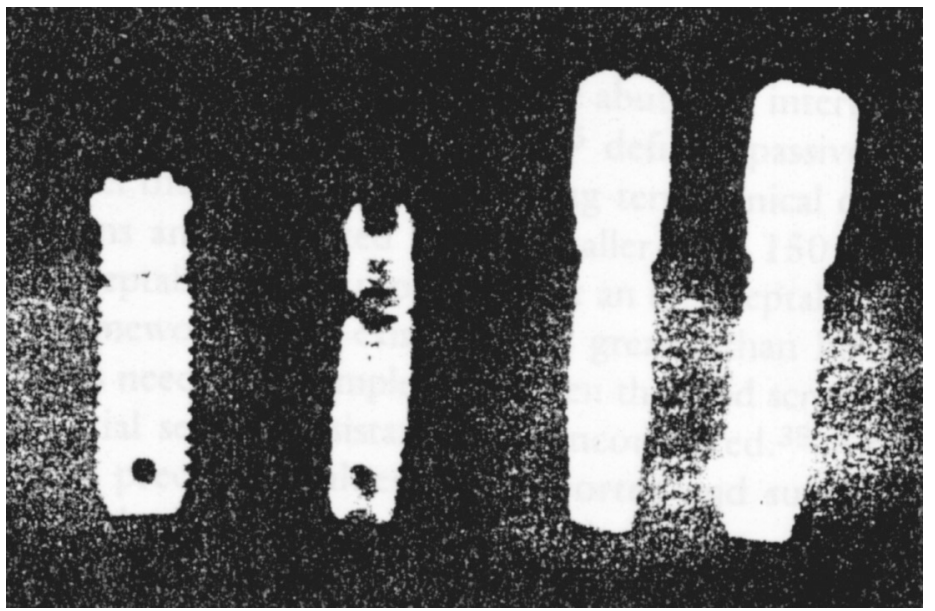

Figure 52: Fatigue fracture. 
Table 5: Probability of 5-year implant survival in four anatomic areas.

\begin{tabular}{|l|c|c|c|c|}
\hline Anatomic area & $\begin{array}{l}\text { 5-year } \\
\text { survival } \\
(\%)\end{array}$ & $\begin{array}{l}\text { 5-year implant } \\
\text { fracture } \\
\text { (probability } \\
\text { of no occur- } \\
\text { rence) }(\%)\end{array}$ & $\begin{array}{l}\text { 5-year screw } \\
\text { loosening } \\
\text { (probability of } \\
\text { no occurrence) } \\
(\%)\end{array}$ & $\begin{array}{l}\text { 5-year screw } \\
\text { fracture } \\
\text { (probability of } \\
\text { no occurrence) } \\
(\%)\end{array}$ \\
\hline Anterior maxilla & 95.7 & 100 & 89.5 & 100 \\
\hline Posterior maxilla & 95.9 & 99.0 & 88.8 & 99.2 \\
\hline Anterior mandible & 96.8 & 100 & 92.7 & 100 \\
\hline Posterior mandible & 94.4 & 97.0 & 83.8 & 96.5 \\
\hline
\end{tabular}

crown (resulting in load eccentricity and major bending overloads) and presence of defects (inclusions, inner porosity) in the material.

A secondary cause of implant loss is the misfunctional behaviour of the components in the superstructure surmounting the fixture. Fatigue fracture of abutment and prosthetic screws or loosening of inner fits are acknowledged occurrences. These failures typically originate in poor tightening of the screws or excessive backlash between mating parts.

Since the market of dental fixtures is chiefly oriented towards private professionals who do not perform long-term follow-ups, a comprehensive statistics of clinical results is not available. Among the wealth of prostheses implanted till now, the observed performance testifies to a better survival rate of osseointegrated implants in comparison with fibro-osseointegrated ones. The recent review by Eckert and Wollan [83] of some one thousand endosseous implants placed for more than 10 years is one of the more complete sources of information in modern implantology. Table 5 collects the probability of 5-year implant survival in four anatomic zones of the jaw emerging from the investigation. Examined causes of failure are implant fracture, screw loosening and screw fracture (see previous page).

Data analysis from this retrospective review demonstrates the following. (1) Implant survival is not dependent on the anatomic location of the implant. (2) Implant loss in the anterior maxilla and mandible occurs early with a steady state of implant survival noted soon after secondstage surgery. (3) Implant loss in the posterior maxilla and mandible occurs over a long period with most of the late implant failure because of implant fracture. (4) Changes in the design of implant components which took place in 1991 have resulted in significant improvements in implant survival in the posterior regions, implant fracture rates and prosthetic complication rates. Continued use of these components is therefore recommended.

\section{References}

[1] Brånemark, P.I., Hansson, B.O. \& Adell, R. et al., Osseointegrated dental implants in the treatment of the edentulous jaw: experience from a 10-year period. Scandinavian Journal of Plastic \& Reconstructive Surgery, 11(Suppl 16), pp. 1-132, 1977.

[2] Wolff, J., Das Gesetz der Transformation der inneren Architektur der Knochen bei patologischen Veränderungen der äusseren Knochenform, Sitz. No 22, 1884.

[3] Rieger, M.R., Adams, W.K. \& Kinzel, G.L., Finite element survey of eleven endosseous implants. Journal of Prosthetic Dentistry, 63, pp. 457-465, 1990. 
[4] Lotz, J.C., Gerhart, Y.N. \& Hayes, W.C., Mechanical properties of metaphysical bone in the proximal femur. Journal of Biomechanics, 24, pp. 317-329, 1991.

[5] Cowin, S.C., Strain assesment by bone cells. Tissue Engineering, pp. 181-186, 1988.

[6] Lakes, R.S. \& Katz, J.L., Viscoelastic properties of wet cortical bone. II. Relaxation and mechanisms. III. A nonlinear constitutive equation. Journal of Biomechanics, 12, pp. 679-698, 1979.

[7] Evans, F.G., Stress and Strain in Bones. Their Relations to Fracture and Osteogenesis, Charles C. Thomas: Springfield, IL, 1957.

[8] Hert, J.A., Liskova, M. \& Landa, J., Reaction of bone to mechanical stimuli. Part 1. Continuous and intermittent loading of tibian rabbit. Folia Morphologica., 19, pp. 290-317, 1971.

[9] Fung, Y.C., Biomechanics: Mechanical Properties of Living Tissues, 2nd edn., Springer: New York, 1993.

[10] Reilly, D.T. \& Burstein, A.H., The elastic and ultimate properties of compact bone tissue. Journal of Biomechanics, pp. 393-405, 1975.

[11] Knets, I.V., Mechanics of biological tissues, a review. Polymer Mech., 13, pp. 434-440, 1978.

[12] Arendts, F.J. \& Sigolotto, C., Standardabmessungen, Elastizitätskennwerte und Festigkeitsverhalten des Human-Unterkiefers, ein Beitrag zur Darstellung der Biomechanik der Unterkiefer - Teil I. Biomed. Technik, 34(10), pp. 248-255, 1989.

[13] Tsai, S.W. \& Wu, E.M., A general theory of strength for anisotropic materials. Journal of Composite Materials, 5, pp. 58-60, 1971.

[14] Natali, A.N. \& Pavan, P.G., Numerical modelling in dental biomechanics, XV AIMETA Congress, Taormina, 26-29-9-2001, cd support, 2001.

[15] Taylor, W.R., Warner, M.D. \& Clift, S.E., Finite element prediction of endosteal ad periosteal bone remodelling in the turke ulna: effect of remodelling signal and dead-zone definition. Journal of Engineering in Medicine, 217, pp. 349-356, 2003.

[16] Tsubota, K., Adachi, T. \& Tomita, Y., Cancellous bone adaptation in proximal femur predicted by trabecular surface remodeling simulation, BED, 50. ASME Conference, pp. 299-300, 2001.

[17] Kerner, J., Huiskes, R., van Lente, G.H., Weinans, H., van Rietbergen, B., Engh, C.A. \& Amis, A.A., Correlation between pre-operative periprosthetic bone density and post-operative bone loss in THA can be explained by strain-adaptive remodelling. Journal of Biomechanics, 32, pp. 695-703, 1999.

[18] Gotfredsen, K., Berglundh, T. \& Linde, J. \& Bone reactions adjacent to titanium implants subjected to static load. Clinical Oral Implants Research, 12, pp. 1-8, 2001.

[19] Branemark, R., Ohrnell, L.-O., Nilsson, P. \& Thomsen, P., Biomechanical charaterization of osseointegration during healing: an experimental in vivo study in the rat. Biomaterials, 18, pp. 969-978, 1997.

[20] Bourauel, C., Numerical simulation of orthodontic tooth movements, $X V$ AIMETA Congress, Taormina, 26-29-9-2001, cd support, 2001.

[21] Cruz, M., Wassall, T., Toledo, E.M., Da Silva Barra, L.P. \& De Catro Lemonge, A.C., Three-dimensional finite element stress analysis of a cuneiform - geometry implant. International Journal of Oral \& Maxillofacial Implants, 18, pp. 675-684, 2003.

[22] Ravaglioli, A. \& Krajewski, A., Bioceramics, Chapman \& Hall: London, 1992.

[23] Kade, H., Akagawa, Y., Hashimoto, M. \& Tenma, H., The effects of a stress-absorbing system involved in the superstructure supported by hydroxyapatite-coated implants in monkeys. Internationl Journal of Oral \& Maxillofacial. Implants, 10(2), pp. 213-219, 1995. 
[24] Lang, L.A., Kang, B., Rui-Feng, W. \& Brien, R.L., Finite element analysis to determine implant preload. Journal of Prosthetic Dentistry, 90, pp. 539-546, 2003.

[25] Bülent, E., Numerical analysis of dental implant system in three-dimension. Advances in Engineering Software, 33, pp. 109-113, 2002.

[26] Merz, B.R. \& Hunenbart, S., Meccanica della connessione impianto-abutment: conicità di $8^{\circ}$ rispetto a una connessione testa a testa. The International Journal of Oral \& Maxillofacial Implants, 2000****.

[27] Hansson, S., A conical implant-abutment interface at the level of the marginal bone improves the distribution of stresses in the supporting bone. Clinical Oral Implants Research, 14, pp. 286-293, 2003.

[28] Vander Sloten, J., Van Oosterwyck, H., Puers, R. \& Naert, I., Finite element studies of the bone adaptive response around functionally loaded oral implants. XV AIMETA Congress, Taormina, 26-29-9-2001, cd Support, 2001.

[29] Menicucci, G., Mossolov, A., Mozzati, M., Lorenzetti, M. \& Preti, G., Tooth-implant connection: some biomechanical aspects based on finite element analysis. Clinical Oral Implants Research, 13, pp. 334-341, 2002.

[30] Heywood, R.B., Designing Against Fatigue, Chapman and Hall Ltd.: London, 1962.

[31] Dragoni, E., Effect of nut geometries on srew thread stress distribution: photoelastic results. Journal of Strain Analysis, 27(1), pp. 1-6, 1962.

[32] Dragoni, E., Effect of thread shape on screw stress concentration by photoelastic measurements. ASME Journal of Offshore Mechanics and Arctic Engineering, 116, pp. 228-232, 1994.

[33] Shigley, J.E., Mechanical Engineering Design, Mcgraw-Hill: New York, 1986.

[34] Peterson, R.E., Stress Concentration Factors, Wiley: New York, 1974.

[35] Juvinall, R.C.E. \& Marsheck, K.M., Fundamentals of Machine Component Design, Wiley: New York, 1991.

[36] Strozzi, A., Costruzione di Macchine, Pitagora Editrice: Bologna, 1998.

[37] Ciavarella, M., Hills, D.A. \& Monno, G., The influence of rounded edges on indentation by a flat punch. Proceedings Insitution of Mechanical Engineers, 212 C, pp. 319-328, 1998.

[38] Arataki, T., Adachi, Y. \& Kishi, M., Two-dimensional finite element analysis of the influence of bridge design on stress distribution in bone tissues surrounding fixture of osseointegrated implants in the lower molar region. Bulletin of Tokyo Dental College, 39(3), pp. 199-209, 1998.

[39] Menicucci, G. et al., Tooth-implant connection:some biomechanical aspects based on finite element analyses. Clinical Oral Implants Research, 13, pp. 334-341, 2002.

[40] Skalak, R., Brunski, J.B. \& Mendelson, M., A method for calculating the distribution of vertical forces among variable-stiffness abutments supporting a dental prosthesis presented at the 1993 bioengineering conference, 25-29 june 1993, breckenridge, co. American Society of Mechanical Engineers, Bioengineering Division, 24, pp. 347-350, 1993.

[41] Mcalarney, M.E. \& Stavropoulos, D.N., Determination of cantilever length-anteriorposterior spread ratio assuming failure criteria to be the compromise of the prosthesis training screw-prosthesis joint. International Journal of Oral Maxillofacial Implants, 11, pp. 331-339, 1996.

[42] Korioth, T.W.P. \& Johann, A.R., Influence of mandibular superstructure shape on implant stresses during simulated posterior biting. Journal of Prosthetic Dentistry, 82, pp. 67-72, 1999.

[43] Mcalarney, M.E. \& Stavropoulos, D.N., Theoretical cantilever lenghts versus clinical variables in fifty-five clinical cases. Journal of Prosthetic Dentistry, 83, pp. 332-343, 2000. 
[44] Nagasao, T., Koboyashi, M., Tsuchiya, Y., Kaneko, T. \& Nakajima, T., Finite element analysis of the stresses around fixtures in various reconstructed mandibular models - part II (effect of horizontal load), Journal of Cranio-Maxillofacial Surgery, 31, pp. 168-175, 2003.

[45] Zarone, F., Apicella, A., Nicolais, L., Aversa, R. \& Sorrentino, R., Mandibular flexure and stress build-up in mandibular full-arch fixed protheses supported by osseointegrated implants. Clinical Oral Implants Research, 14, pp. 103-114, 2003.

[46] Inou, N., Iioka, Y., Fujiwara, H. \& Maki, K., Functional adaptation of mandibular bone. In: Hayashi, K. \& Ishikawa, H. (eds.), Computational Biomechanics, Springer-Verlag: Tokyo, pp. 23-42, 1996.

[47] Jaecques, S.V.N., Van Oosterwyck, H., Muraru, L., Van Cleynenbreugel, T., De Smet, E., Wevers, M., Naert, I. \& Vander Sloten, J., Individualised micro CT-based finite element modelling as a tool for biomechanical analysis related to tissue engineering of bone. Biomaterials, 25(9), pp. 1683-1696, 2004.

[48] Geng, J.P., Tan, K.B.C., Gui- Rong, Liu, Application of finite element analysis in implant dentistry: A review of literature. Journal of Prosthetic Dentistry, 85(6), pp. 585-598, 2001.

[49] Miles, A.W. \& Tanner, K.E., Strain Measurement in Biomechanics, Chapman \& Hall: London, 1992.

[50] Orr, J.F., Humphreys, P.K., James, W.V. \& Bahrani, A.S., The application of photoelastic techniques in orthopaedic engineering. Applied Stress Analysis. Hyde, T.H. \& Ollerton, E. (eds.), Barking: Elsevier, 1990.

[51] Patra, A.K., DePaolo, J.M., D’Souza, K.S., DeTolla, D. \& Meenaghan, M.A., Guidelines for analysis and redesign of dental implants. Implant Dentistry, 7(4), pp. 355-367, 1998.

[52] Van, R., Odgaard, A., Kabel, J. \& Huiskes, R., Direct mechanics assessment of elastic symmetries and properties of trabecular bone architecture. Journal of Biomechanics, 29(12), pp. 1653-1657 B, 1996.

[53] Liu, Y.J., On the simple-solution method and non-singular nature of the BIE/BEMa review and some new results. Engineering Analysis with Boundary Elements, 24, pp. 789-795, 2000.

[54] Armentani, E., Caputo, F., Citarella, R., Esposito, R., Infante, M. \& Lavorgna, G., Sulla valutazione numerica delle condizioni elastostatiche di un impianto dentario endosseo, XXIX AIAS Congress, 6-9/7/2000, Lucca: Italy, 2000.

[55] Armentani, E., Calì, C., Caputo, F., Citarella, R., Infante, M. \& Lavorgna, G., Modello agli elementi di contorno per l'analisi tensionale dell'articolazione temporo-mandibolare., XXXI AIAS Congress, 18-21/7/2002, Parma: Italy, 2002.

[56] Tonti, E., Un semplice metodo numerico: il metodo delle celle. Atti del VII Convegno Italiano di Meccanica Computazionale, Trieste: Italy, 1993.

[57] Tonti, E., A direct discrete formulation of field laws: the cell method. Computer Modeling in Engineering \& Science, 2(2), pp. 237-258, 2001.

[58] Cosmi, F., Numerical Modeling of porous materials' mechanical behaviour with the cell method, Proc. of Second MIT Conference on Computational Fluid and Solid Mechanics, Massachusetts Institute of Technology, Cambridge, MA, U.S.A, 17-20.06.2003, pp. 1915-1917, 2003.

[59] Cosmi, F. \& Dreossi, D., Evaluation of a new index of bone structure load bearing capability. EuroPACS-MIR 2004 Conference, Trieste: Italy, 16-19-9-2004, 2004.

[60] Cosmi, F., Numerical solution of plane elasticity problems with the cell method. Computer Methods in Engineering \& Sciences, 2(3), pp. 1-7, 2001. 
[61] Fessler, H., Load distribution in a model of a hip joint. Journal of Bone Joint Surgery, 39, pp. 143-153, 1957.

[62] Noonan, M.A., The use of photoelasticity in the study of cavity prepaight586 strain gauges. Engng rations, Journal of Dentistry for Children, 16, pp. 24-28, 1949.

[63] Granath, L.E., Further photoelastic studies on the restorations between the cavity and occlusal portion of Class 2 restorations. Odent. Review, 15, pp. 290-298, 1964.

[64] El-Abrashi, M.K., Craig, R.G. \& Peyton, F.A., Experimental stress analysis of dental restorations Part 7. Structural design and stress analysis of fixed partial dentures. Journal of Prosthetic Dentistry, 23, p. 177, 1970.

[65] Farah, J.W., MacGregor, A.R. \& Miller, T.P.G., Stress analysis of disjunct removable partial dentures. Journal of Prosthetic Dentistry, 42, pp. 271-275, 1979.

[66] Atmaram, G.H. \& Mohammed, H., Photoelastic stress analysis of dental implants with different root configurations. New York State Dental Journal, 47, pp. 30-33, 1981.

[67] Little, E.G., Experimental stress analysis of the Geomedic Knee joint using embedded strain gauges. Engineering Medicine, 14(2), pp. 69-74, 1985.

[68] Little, E.G., Daly, G., Power, B., Dawson, M. \& Kelly, P., The development of a model for the investigation of stresses in the cement layer underlying a tibial plateau. Strain, pp. 19-25, 1987.

[69] Prati, E. \& Freddi, A., A critical evaluation of some current experimental models simulating the human knee joint. Proc 7th Int. Cong. of Biomechanics, Warsaw, Poland, pp. 236-242, 1981.

[70] Pezzoli, M.D., Rossetto, M. \& Calderale, P.M., Evaluation of load transmission by distal-extension removable partial dentures by using reflection photoelasticity. Journal of Prosthetic Dentistry, pp. 329-337, 1986.

[71] Calderale, P.M., Rossetto, M., Pezzoli, M. \& Franciosi, A., A photoelastic study of the biomechanical behaviour of dental implants, XI Polish-Italian Seminar, 20-21/4/1993, Turin, Italy, pp. 75-82, 1993.

[72] Celasco, M., Calcagno, L., Pezzoli, M., Modica, R., Rossetto, M. \& Calderale, P.M., Valutazione della trasmissione di carichi protesici mediante tecniche fotoelastiche ed olografiche. Quintessenze International, 6/7, pp. 433-438, 1993.

[73] Chun, H.J., Cheong, S.Y., Han, J.H., Heo, S.J., Chiung, J.P., Rhyu, I.C., Choj, Y.C., Baik, H.K., Ku, Y. \& Kim, M.H., Evaluation of design parameters of osseointegrated dental implants using finite element analysis. Journal of Oral Rehabilitation, 29, pp. 565-574, 2002.

[74] Holmgren, E.P., Seckinger, R.J., Kilgren, L.M. \& Mante, F., Evaluating parameters of osseointegrated dental implants using finite element analysis: a two dimensional comparative study examining the effects of implant diameter, implant shape, and load direction. Journal of Oral Implantology, 24, pp. 80-88, 1998.

[75] Bianchi, A., Implantologia e Implantoprotesi, UTET: Milano, 1999.

[76] Lekholm, U., Adell, R. \& Brhanemark, P.I., Complications. In: Osseointegration in Clinical Dentistry, Quintessence: Chicago, pp. 233-340, 1985.

[77] Merz, B.R., Hunenbart, S. \& Belser, U.C., Meccanica della connessione impiantoabutment: conicità di $8^{\circ}$ rispetto a una connessione testa a testa. Quintessenze International, 3/4, pp. 111-119, 2001.

[78] Clienti, C., Grasso, G. \& Pulvirenti, G., Analisi delle capacità di smorzamento delle sollecitazioni trasmesse all'osso da un nuovo impianto dentale, AIAS Congress, Alghero, 12-15/9/2001, pp. 41-49, 2001. 
[79] Iplikcioglu, H. \& Akca, K., Comparative evaluation of the effect of diameter, length and number of implants supporting three-unit fixed partial prosthese on stress distribution in the bone. Journal of Dentistry, 30, pp. 41-46.

[80] Van Oosterwyck, H., Vander Sloten, J., De Cooman, M., Lievens, S., Puers, B., Duyck, J. \& Naert, I., Bone overload versus underload: Determinant factors in the long-term success of oral implants. Journal of Biomechanics, 31(Suppl 1), p. 42, 1998.

[81] Akagawa, Y., Sato, Y., Teixeira, E.R., Shindoi, N. \& Wadamoto, M.W., A mimic osseointegratioed implant model for three-dimensional finite element analysis. Journal of Oral Rehabilitation, 30, pp. 41-45, 2003.

[82] Strozzi, A., Vaccari, P., Bruzzesi, G., De Pol, A. \& Zaffe, D., Analisi tensionale di impianti dentari: studio di forme tradizionali e nuove proposte. V Giornata di studio su "Biomateriali e loro applicazioni in Biomeccanica-Sistemi biomeccanici ed interazione con i tessuti biologici”. Catania 6 Luglio 2001.

[83] Eckert, S. \& Wollan, P., Retrospective review of 1170 endosseous implants placed in partially edentulous jaws. Journal of Prosthetic Dentistry, 79(4), pp. 415-421, 1998. 